\title{
Paving Spin-Wave Fibers in Magnonic Nanocircuits Using Spin-Orbit Torque
}

\author{
Xiangjun Xing, ${ }^{1,2}$ Philip W. T. Pong, ${ }^{2}$ J. Åkerman, ${ }^{3,4}$ and Yan Zhou, \\ ${ }^{1}$ School of Physics and Optoelectronic Engineering, Guangdong University of Technology, \\ Guangzhou 510006, China \\ ${ }^{2}$ Department of Electrical and Electronic Engineering, The University of Hong Kong, Hong Kong, China \\ ${ }^{3}$ Department of Physics, University of Gothenburg, Fysikgränd 3, 41296 Gothenburg, Sweden \\ ${ }^{4}$ Material and Nano Physics, School of ICT, KTH Royal Institute of Technology, 16440 Kista, Sweden \\ ${ }^{5}$ School of Science and Engineering, The Chinese University of Hong Kong, Shenzhen 518172, China \\ (Received 20 September 2016; revised manuscript received 31 March 2017; published 24 May 2017) \\ Recent studies reveal that domain walls in magnetic nanostructures can serve as compact, energy-efficient \\ spin-wave waveguides for building magnonic devices that are considered promising candidates for \\ overcoming the challenges and bottlenecks of today's CMOS technologies. However, imprinting long \\ strip-domain walls into magnetic nanowires remains a challenge, especially in bent geometries. Here, through \\ micromagnetic simulations, we present a method for writing strip-domain walls into bent magnetic nanowires \\ using spin-orbit torque. We employ $\mathrm{Y}$-shaped magnetic nanostructures as well as an $\mathrm{S}$-shaped magnetic \\ nanowire to demonstrate the injection process. In addition, we verify that the $Y$-shaped nanostructures that \\ incorporate strip-domain walls can function as superb spin-wave multiplexers and that spin-wave propagation \\ along each conduit can be controllably manipulated. This spin-wave multiplexer based on strip-domain walls \\ is expected to become a key signal-processing component in magnon spintronics.
}

DOI: 10.1103/PhysRevApplied.7.054016

\section{INTRODUCTION}

Magnonic technologies [1-4] have great potential to overcome the obstacles met in CMOS technologies $[5,6]$; for example, magnonic devices can provide enhanced throughput of information and reduced power consumption [3,7]. Additionally, magnonic circuits are more suitable for implementing non-Boolean computations $[8,9]$ for special data processing such as pattern recognition [10]. Controllable, energy-efficient spin-wave propagation in magnetic nanostructures is a crucial step toward practical magnonic nanodevices. In experimentally demonstrated prototype magnonic devices [11-14], the Damon-Eshbach propagation geometry has been widely used because of the high group velocity and excitation efficiency of the related spin waves $[3,15]$. Highly tunable spin waves and spin-wave beams can be excited using spin-transfer torque [16-18]. Recently, Topp et al. [19] and Duerr et al. [20] presented a procedure for forming a self-cladding magnonic waveguide with ultranarrow internal channels, which can indeed enhance spin-wave transmission but cannot be applied to bent magnetic wires $[19,20]$. Later, Vogt et al. $[11,21]$ demonstrated continuous spin-wave propagation along either conduit of a magnonic multiplexer by applying an electric current along a buried metallic layer. Both approaches require an applied force, either an electric current $[11,21]$ or a magnetic field $[19,20]$, to

zhouyan@cuhk.edu.cn maintain the wave-guiding channels, with a considerable associated energy cost.

Alternatively, Garcia-Sanchez et al. [22] have proposed using elongated domain walls to guide spin waves, and

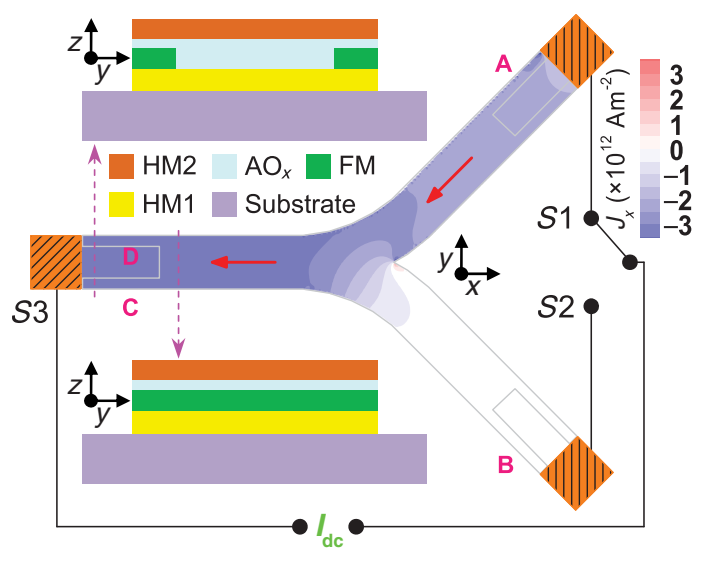

FIG. 1 Device structure and control circuit. The Y-shaped nanostructure is patterned from a multilayer film, HM1/ $\mathrm{FM} / \mathrm{AO}_{x} / \mathrm{HM} 2$. Each end of the FM layer has two narrow pads $150 \mathrm{~nm}$ long and $20 \mathrm{~nm}$ wide for nucleating seed domain walls. A switch connects leads $S 1-S 3$ and $S 2-S 3$ to the direct-current source $I_{\mathrm{dc}}$. The current is confined in the HM2 layer by the insulating $\mathrm{AO}_{x}$ layer. The current profile between $S 1-S 3$ overlaid on the FM layer exhibits an inhomogeneity at the transition region because of the variation in the wire width. $J_{x}$ is the $x$ component of the current density, and the red arrows denote inplane current directions. Here, the opening angle between the two arms, symmetrical relative to the horizontal base, is $90^{\circ}$. 

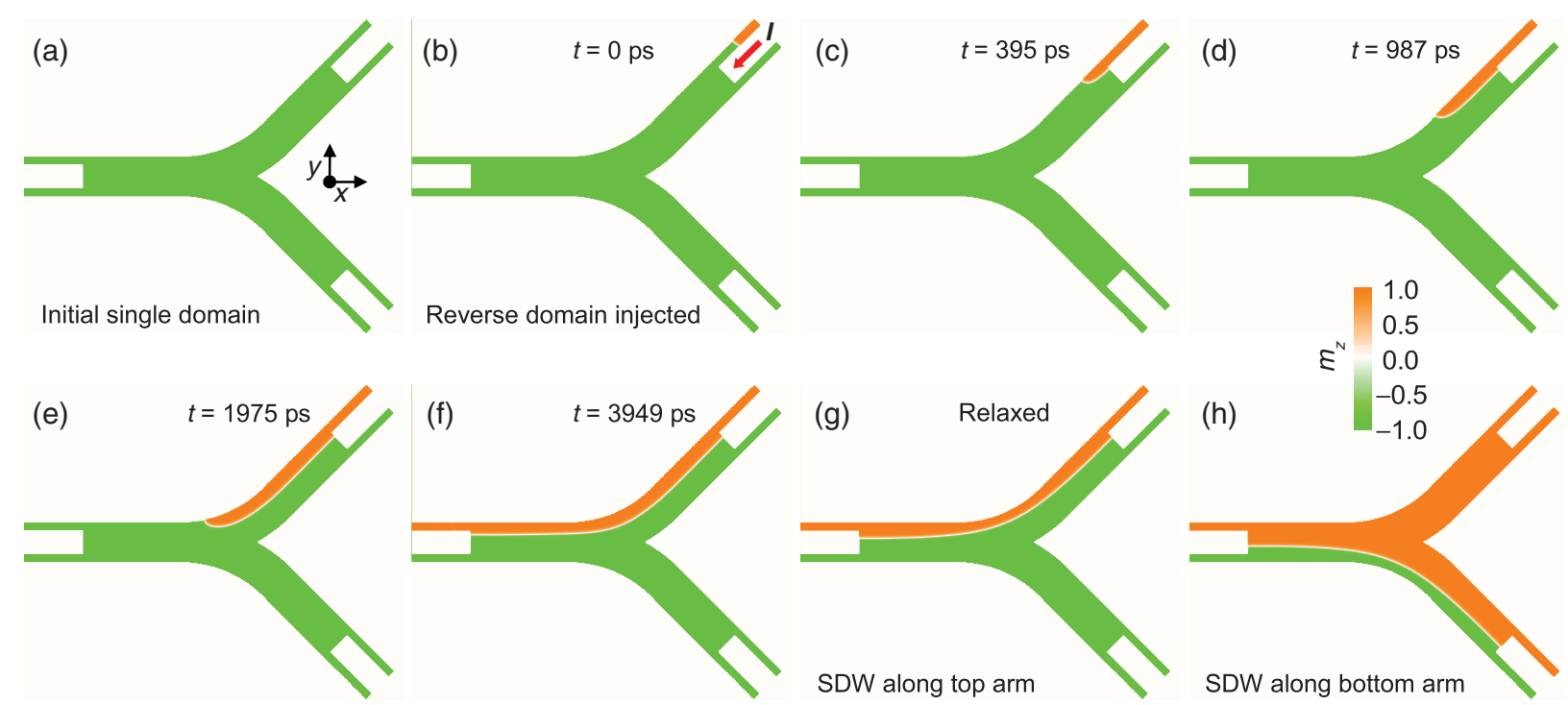

FIG. 2 SDW-injection process starting from a right seed domain. (a) Initial single-domain state. (b) Seed reverse domain formed at nucleation pad $A$. Current is switched on at 0 ps when the seed domain is just formed. (c)-(f) Transient-state SDWs at indicated times after current application. (g) Static SDW after relaxation from 3949 ps. (h) Static SDW in the bottom conduit. The complete dynamic process is shown in the Supplemental Material [27] Video S1(a).

they have numerically demonstrated spin-wave channeling along a bent wire. Further, Xing and Zhou [23] presented a method for reliably writing a domain wall into a magnetic wire with the Slonczewski spin-transfer torque. Most recently, Wagner et al. [24] experimentally verified the feasibility of employing magnetic domain walls as spin-wave channels. Under this scheme, the decay of spin waves due to boundary scattering and intermodal scattering can be reduced, and, moreover, no additional energy needs be consumed to maintain the channels after formation. Thus, this waveguide scheme appears very promising for magnonic devices [25]. However, injecting a long
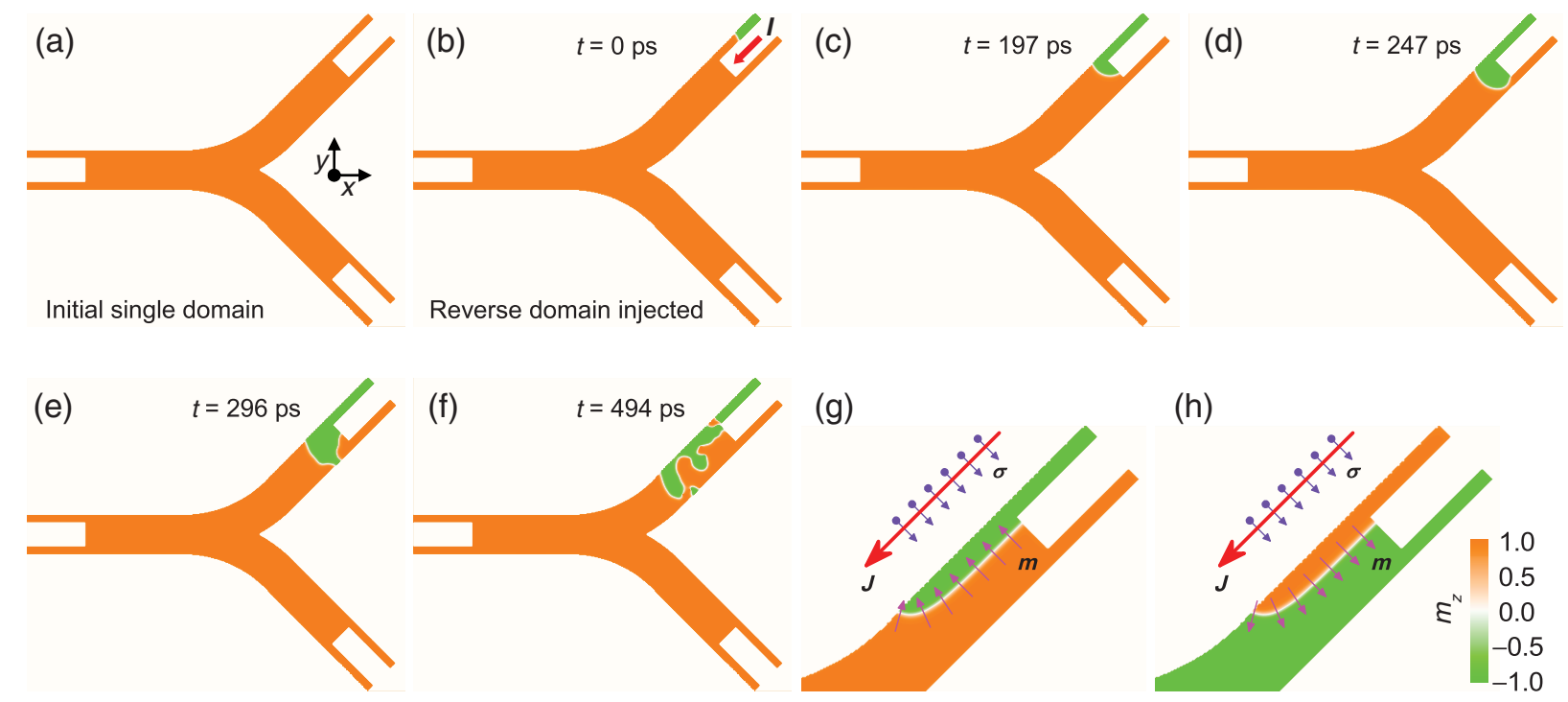

FIG. 3 Injection process initiated from a wrong seed domain and microscopic origin of SDW stabilization and destabilization. (a) Initial single-domain state. (b) Seed domain formed at nucleation pad $A$. Current is switched on at 0 ps when the seed domain just forms. (c)-(f) Transient states at indicated times after current application. No SDW can be written into the arm because of chaotic dynamics. The complete dynamic process is shown in Supplemental Material [27] Video S1(b). (g) Spin orientation in the ridge of the fictitious SDW is opposite the electrons' spin orientation set by the SHE [28], and, therefore, the SDW ridge will be destabilized by SHT, leading to disordered domain patterns. (h) Spin orientation in the ridge of the right SDW is parallel to the electrons' spin orientation defined by the SHE, and, thus, no SHT acts on the SDW ridge. The SDW head feels a SHT and moves forward. 

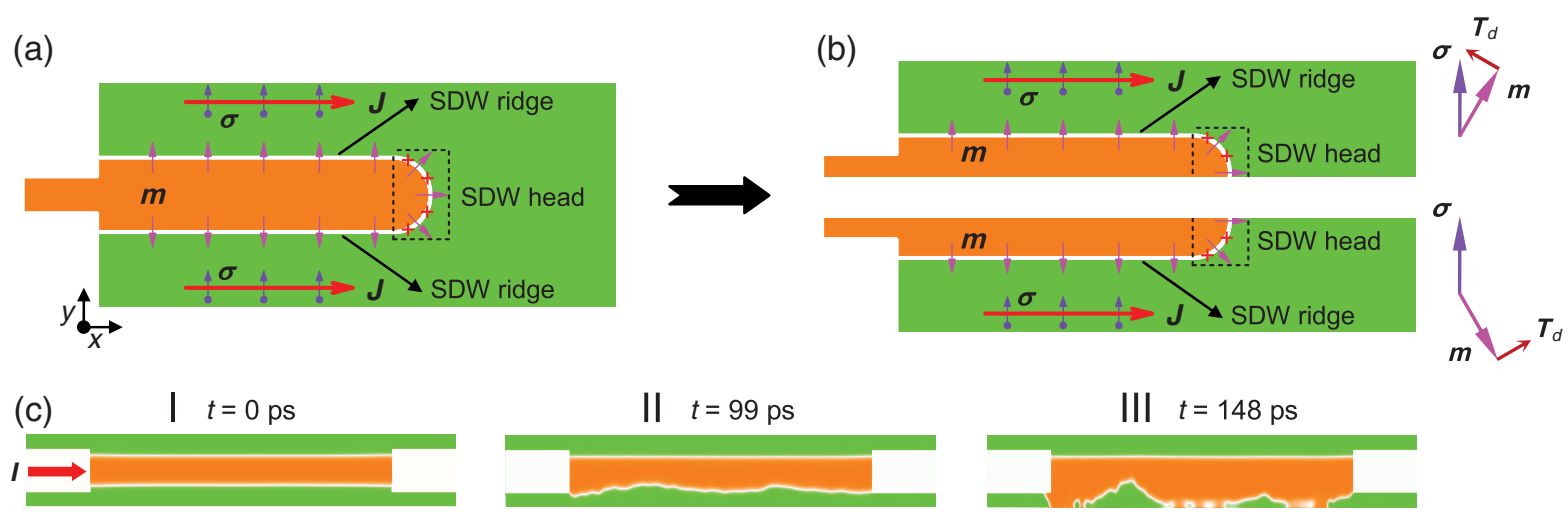

(d)
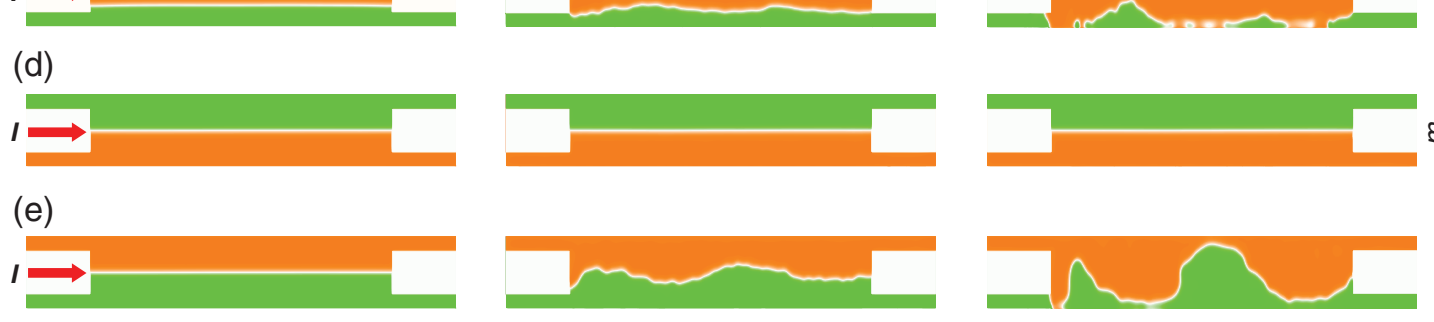

(e)

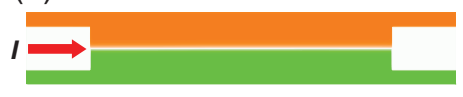

FIG. 4 A physical mechanism responsible for domain-wall stabilization and destabilization. (a) A strip domain pinned at the left side of a wire has two paired SDW ridges (linear) and a SDW head (semicircle) that form a half-Skyrmion with finite topological charge (denoted by "+"). J is the current density, $\boldsymbol{\sigma}$ stands for the electrons' spin orientation given by the SHE, and $\mathbf{m}$ represents the magnetization direction in the domain wall. (b) The split strip domains, each with a single SDW: the linear SDW ridge is parallel to the edge, and the bent SDW head (surrounded by the box) is attached to the edge, forming a quarter-Skyrmion. Here, the SDW head still has nonzero topological charge. The rightmost schematics depict typical alignments of $\mathbf{m}$ with respect to $\boldsymbol{\sigma}$ and the associated SHT ( $\mathbf{T}_{d}$ ) indicating that once $\mathbf{m}$ is misaligned from $\pm \boldsymbol{\sigma}$, a finite SHT arises which tends to drag $\mathbf{m}$ toward $\boldsymbol{\sigma}$. Dynamics of (c) the paired SDWs, (d) the isolated SDW where $\mathbf{m} / / \boldsymbol{\sigma}$, and (e) the isolated SDW where $\mathbf{m}$ is antiparallel to $\boldsymbol{\sigma}$, under the current-induced SHT. Zero ps corresponds to the time when the current is applied to the equilibrium spin configuration.

strip-domain wall (SDW) [26] into a bent magnetic wire remains elusive. The domain-wall channels in the bent wires in Ref. [22] were preset via numerical conjectures; the domain-wall injection procedure proposed in Ref. [23] and that adopted in Ref. [24] seem valid only for straight magnetic wires. To allow broader applicability of the waveguide scheme, a procedure for writing long SDWs into bent magnetic wires is urgently needed.

In this work, we demonstrate how to form a long SDW in bent magnetic nanostructures (Figs. 1-3) by using the emergent spin-orbit torque (SOT) [29-33]. We stress that SOT resulting from the spin Hall effect (SHE) possesses the correct symmetry (i.e., both the magnetization and electron spin orientation rotate locally with position along the length of a bent wire, but the resulting torque is always orthogonal to the length) to push the SDW head when stabilizing the SDW ridge (Fig. 4) and that a stable SDW cannot be realized using the scheme experimentally established in Ref. [32] because of some intrinsic physical restrictions. Compared to the approach developed in Ref. [23], the present method can be applied to magnetic wires with various geometries and to magnetic wires made of a magnetic insulator. The procedure has been used to generate SDW-based spin-wave channels in Y-shaped nanostructures [11] and also in an S-shaped magnetic wire to show its robustness. This research should boost the popularity of magnonic devices using domain walls as spin-wave channels.

\section{MATERIALS AND DEVICE STRUCTURE}

The basic structure of the domain-wall injection is shown in Fig. 1. The main body is a Y-shaped nanostructure (middle panel, Fig. 1) made of the ultrathin multilayered films $\mathrm{HM} 1 / \mathrm{FM} / \mathrm{AO}_{x} / \mathrm{HM} 2$ (bottom panel, Fig. 1), where FM is a ferromagnetic layer with perpendicular magnetic anisotropy (PMA), HM represents heavy-metal layers with strong spin-orbit coupling, and $\mathrm{AO}_{x}$ is an oxide layer that insulates the FM layer from the HM2 layer [34]. The asymmetric interfaces of the FM layer combined with the strong spin-orbit coupling in the HM1 layer can induce a large interfacial Dzyaloshinskii-Moriya interaction (IDMI) in the ferromagnet [39], which is required to stabilize SDWs in bent geometries. Narrow pads $(A, B, C$, and $D)$ for domain-wall nucleation at each end of the FM layer (top panel, Fig. 1) are used to form seed reverse domains. An inplane charge current flowing in the HM2 layer along $S 1-S 3$ or $S 2-S 3$ generates a pure transverse spin current in the perpendicular direction via the spin Hall effect [28,40], which, in turn, exerts spin-orbit torques on the magnetization in the FM layer [41]. We intentionally insert a thin $\mathrm{AO}_{x}$ layer between the FM layer and $\mathrm{HM} 2$ layer to prevent 
charge current from flowing into the ferromagnet, thus, eliminating the Zhang-Li spin-transfer torques (STTs) $[42,43]$ in the ferromagnet [44]. Depending on the current path used ( $S 1-S 3$ or $S 2-S 3$ ), a SDW can be written into the top or bottom arm.

\section{MICROMAGNETIC MODEL AND SIMULATIONS}

We perform micromagnetic simulations to study SDW injection and spin-wave propagation along the SDWs in Y-shaped nanostructures by numerically solving the modified Landau-Lifshitz-Gilbert equation [45,46] with spin-orbit torques [41,47],

$$
\partial \mathbf{m} / \partial t=-\gamma\left(\mathbf{m} \times \mathbf{H}_{\mathrm{eff}}\right)+\alpha(\mathbf{m} \times \partial \mathbf{m} / \partial t)+\mathbf{T}_{d}+\mathbf{T}_{f},
$$

where $\mathbf{m}=\mathbf{M} / M_{s}$ is the unit vector along the magnetization $\mathbf{M}$, and $M_{s}$ is the saturation magnetization. $\mathbf{H}_{\text {eff }}=$ $-\left(1 / \mu_{0}\right) \delta E / \delta \mathbf{M}$ is the effective field in the ferromagnet with $\mu_{0}$ denoting the vacuum permeability, and $E=E_{d}+$ $E_{u}+E_{x}+E_{\mathrm{DM}}+E_{Z}$ the total energy density, including the magnetostatic, anisotropy, exchange, IDMI, and Zeeman energy contributions. $\mathbf{T}_{d}$ and $\mathbf{T}_{f}$ represent the dampinglike [48] and fieldlike [49] torques, respectively.

The LLG micromagnetic simulator [50] is used to implement all the simulations, in which only the FM and HM2 layers are explicitly incorporated, as in Ref. [46]. We do not directly include the $\mathrm{HM} 1$ and $\mathrm{AO}_{x}$ layers in the simulations, but, instead, we simply take account of their physical effects, that is, the IDMI caused by the HM1 layer and the insulation of the FM layer from the HM2 layer enabled by the $\mathrm{AO}_{x}$ layer. Both the FM and $\mathrm{HM} 2$ layers are $d_{\mathrm{FM}}=d_{\mathrm{HM} 2}=1 \mathrm{~nm}$ in thickness. The width of the wire for all samples is $w=100 \mathrm{~nm}$, and the wire length varies with sample geometry. We examine the SDW-injection process and its stability over a wide range of values of $M_{s}, K_{u}$, and $D$ to account for the sensitivity of the material parameters to interface properties and layer thickness $[38,51,52]$. The results are based on the following material parameters unless otherwise specified: $M_{s}=580 \mathrm{kA} \mathrm{m}^{-1}$, exchange stiffness $A=15 \mathrm{pJ} \mathrm{m}^{-1}$, perpendicular magnetocrystalline anisotropy $K_{u}=$ $0.8 \mathrm{MJ} \mathrm{m}^{-3}$, IDMI strength $D=2.0 \mathrm{~mJ} \mathrm{~m}^{-2}$, and Gilbert damping constant $\alpha=0.02$. These parameters correspond to the experimental values reported for $\mathrm{Pt} / \mathrm{Co} / \mathrm{AlO}_{x}$ [53], $\mathrm{Pt} /(\mathrm{Co}, \mathrm{Fe}) \mathrm{B} / \mathrm{MgO}$ [33], and $\mathrm{Ta} /(\mathrm{Co}, \mathrm{Fe}) \mathrm{B} / \mathrm{TaO}_{x}[35,54]$ systems. For computation, each sample is divided into regular meshes of $2 \times 2 \times 1 \mathrm{~nm}^{3}$ in size, which is much smaller than the exchange length $l_{\mathrm{ex}}=\sqrt{2 A / \mu M_{s}^{2}} \approx$ $8.4 \mathrm{~nm}$ (i.e., the maximum length within which the magnetization can be kept uniform by the short-range exchange interaction), and open boundary conditions are used. For simulations with spin-orbit torques, the spin Hall angle is assumed to be $\Phi_{H}=0.13$ [55], the spin polarization of the carriers in the FM layer $P=0.4$, and a series of values of the Rashba parameter $\alpha_{R}$ are considered $[56,57]$ to examine the influence of the Rashba torque (RT). We consider that the SHE contributes only to the dampinglike torque, while the Rashba effect induces only the fieldlike torque, as implemented in Ref. [46]. The largest Rashba parameter examined gives a Rashba torque 1.5 times as strong as the spin Hall torque (SHT) for $J_{\mathrm{FM}}=J_{\mathrm{HM} 2}$. To check the contribution of the Zhang-Li torques in the SDW injection driven by the SHE, equal current densities in the FM and HM2 layers, i.e., $J_{\mathrm{FM}}=$ $J_{\mathrm{HM} 2}$ and $\beta=\alpha(\beta$ is the nonadiabaticity factor $[42,43])$ are implemented in corresponding simulations. The effect of pinning centers on the SDW injection is examined by considering unrealistically high concentrations $(1 \%$ and $5 \%$ ) of impurities inside the FM layer, which are modeled as randomly distributed sites over the simulation space with $D_{\text {(impurity) }}=-D_{\text {(nonimpurity) }}[33,58]$. We also include a Langevin random field in the effective field to test the thermal-fluctuation effects on the SDW injection.

An electric current $I_{\mathrm{dc}}$ is fed to $S 1-S 3$ or $S 2-S 3$ of the $\mathrm{Y}$-shaped nanostructure. Note that the electric current is confined to the HM2 layer when the oxide layer is present; thus, $J_{\mathrm{HM} 2}=I_{\mathrm{dc}} / w d_{\mathrm{HM} 2}$. The real current distribution along $S 1-S 3$ for $I_{\mathrm{dc}}=300 \mu \mathrm{A}$ is overlaid on the top view in Fig. 1 as a contour plot coded into $J_{x}$ (the $x$ component of the current density), indicating that the current distribution is slightly inhomogeneous around the transition region. To mimic experimental conditions, such real current distributions [58] are used in all of our simulations instead of uniform ones $[59,60]$, and the Oersted fields given by these current distributions are also incorporated in all relevant simulations.

\section{RESULTS}

\section{A. Injection process of a strip-domain wall}

Writing SDWs into magnetic nanostructures is the main focus of the present research. Figure 2 shows the steps for imprinting a SDW. First, a quasiuniform single domain [Fig. 2(a)] is generated, which can be realized numerically by relaxing an artificial spin configuration with $m_{z}=-1$ to static equilibrium. Experimentally, a sufficiently strong magnetic field along $-z$ can be used to set up such a spin configuration. Second, a seed domain wall is injected into the narrow pad $A$. To do this numerically, we apply a sinusoidal field pulse [59] $\mathbf{H}_{i}=\mathbf{H}_{0} \sin \left(2 \pi f_{i} t\right)$ with $\mathbf{H}_{0}=$ $1.4142 \mathrm{~T}\left(\mathbf{H}_{0}\right.$ is in the plane normal to the pad length and $45^{\circ}$ away from the $x-y$ plane), $f_{i}=2.5 \mathrm{GHz}$, and $t=$ 200 ps to the outmost $50 \times w_{N} \mathrm{~nm}^{2}$ of pad $A$ ( $w_{N}$ is the pad width). Figure 2(b) shows the spin configuration at a time when $\mathbf{H}_{i}$ runs for 200 ps. It is worth noting that experimentally, a seed reverse domain can be injected by using a local spin valve formed on the pad [61]. Third, a direct current $\left(I_{\mathrm{dc}}\right.$ in Fig. 1$)$ is applied to the top conduit from 
$S 1$ to $S 3$, and as a result, a transverse vertical spin current is injected into the FM layer. This spin current imposes a spin torque on the seed domain wall via the SHE [28]. The Rashba effect may occur here but will not significantly affect the SDW injection if the relative strength of the RT to SHT is below 0.5; see Appendix A.

The SHT drives the seed domain wall to move forward and enter the arm [Fig. 2(c)]. After entry, the seed domain wall, initially aligning along the transverse direction [Fig. 2(b)], evolves into a SDW with its ridge aligning along the arm [Fig. 2(d)]. It is intriguing that the SHT moves only the head of the SDW, but it does not shift the ridge of the SDW. We show that this is because the magnetization in the SDW ridge (at the SDW head) is parallel (perpendicular) to the electrons' spin orientation defined by the SHE and, therefore, feels a vanishing (considerable) torque. At approximately 1975 ps, the SDW head passes through the bent section of the top conduit and enters into the base. At approximately 3949 ps, the SDW head reaches the left end, and an intact SDW is written into the top conduit. Next, by withdrawing the electric current and relaxing the whole system to static equilibrium, a stable SDW is obtained, as shown in Fig. 2(g). Similarly, a SDW can be imprinted on the bottom conduit from pad $B$ [Fig. 2(h)].

In Fig. 2, the initial domain is downward, the seed reverse domain is upward, and an electric current along $S 1-S 3$ successfully writes a SDW into the top conduit. We now begin the process from an upward initial domain [Fig. 3(a)]: clearly, we need to create a seed domain with downward magnetization [Fig. 3(b)] using a field pulse. Once a seed domain (and then a seed domain wall) forms, an electric current along $S 1-S 3$ is turned on. Figures 3(c)3 (f) illustrate the dynamic evolution of the seed domain wall after the application of current; this is very chaotic and, hence, is totally different from the process in Figs. 2(c)-2(f). In this case, the seed domain wall can enter into the arm [Fig. 3(c)], as in Fig. 2(c), but it cannot sustain a profile as a SDW [compare Figs. 2(d) and 3(d)]. In fact, the early domain wall in Fig. 3(c) already extends transversely and soon contacts the other edge of the arm [Fig. 3(e)]. Thereafter, disordered domain patterns [Fig. 3(f)] appear and evolve dynamically in the arm [see the Supplemental Material [27] Video S1(b)] until the current is turned off. Figures 3(c)-3(f) indicate that it is impossible to inject a SDW into the $\mathrm{Y}$-shaped nanostructure from a downward seed domain situated in $\operatorname{pad} A$.

In Figs. 2 and 3, pad $A$ is used to inject a SDW. SDW injection can, in fact, be initiated from any one of pads $A-D$. However, as in the case using pad $A$, only the seed domain with a specific magnetization orientation can result in the successful imprinting of a SDW, and the seed domain with an opposite orientation will cause chaotic dynamics [see the Supplemental Material [27] Videos S1(a)-S1(h) for details]. The dependence of SDW injection on the magnetization orientation of a seed domain can be understood as follows: assuming that a SDW can also be injected into the arm from pad $A$ with the wrong magnetization orientation [as shown in Fig. 3(b)], the magnetization orientation in the ridge of the SDW will be antiparallel to the spin orientation of the electrons polarized through the SHE [see Fig. 3(g)]. However, only if the magnetization in the ridge of a SDW is parallel to the spin orientation of the polarized electrons [see Fig. 3(h)] can the striplike profile of the SDW be maintained, and the SDW continues to exist in the arm [Fig. 4(d)]; otherwise, the striplike profile cannot be dynamically stabilized and disordered domain patterns form instead [Fig. 4(e)]. This picture is systematically corroborated in Fig. 4.

\section{B. Current-driven dynamics of strip-domain walls}

Figure 4(a) shows a strip domain (pinned at the left end of the wire) that is enclosed by two parallel SDW ridges and a SDW head (forming a half-Skyrmion also called a meron $[59,62])$. The pinned strip domain with paired SDWs will split into two strip domains [Fig. 4(b)], each of which has an isolated SDW ridge parallel to the edge and a SDW head (as a quarter-Skyrmion) touching the edge if the wire is cut along its middle line. Using stripe domains as the seeds, current-driven generation of Skyrmion bubbles was elegantly demonstrated in the recent literature [32], and the spatially divergent current across the paired SDWs induced by the constriction was reckoned to be responsible for the Skyrmion generation. However, according to a latest theory especially developed to explain the experimental results in Ref. [32], Lin [63] revealed that a strip domain with paired SDWs cannot sustain and exist in the system once a current is applied because the half-Skyrmions (acting as the SDW head) at the ends of a stripe domain will move transversely due to the Magnus force associated with the finite topological charge of a half-Skyrmion. Consequently, a strip domain subjected to a current will be distorted in a zigzag manner and eventually break into massive Skyrmions.

Therefore, inherently, the strip domain with paired chiral SDWs [as shown in Fig. 4(a)] cannot be written into a magnetic wire by current-induced SHT because of the physical limit identified by Lin [63]. Below, we show that there exists the other intrinsic mechanism that makes it impossible to inject such a strip domain with paired chiral SDWs into a wire by SHT (Appendix C, Fig. 13). The reason is that one of the two chiral SDWs has its magnetization antiparallel to the electrons' spin orientation defined by the SHE [as shown in Fig. 4(a)] and will be destabilized by the resulting SHT immediately after the current application. When all the $\mathbf{m}$ vectors inside a SDW are strictly parallel [top panel, Fig. 4(b)] or antiparallel [bottom panel, Fig. 4(b)] to the $\boldsymbol{\sigma}$ vector, the SHT is zero as expected from the expression $\mathbf{T}_{d}=-\gamma \tau_{H}(\mathbf{m} \times \boldsymbol{\sigma} \times \mathbf{m})$, where $\tau_{H}=h J \Phi_{H} / 2 e \mu_{0} M_{s} d_{\mathrm{FM}}, \quad \boldsymbol{\sigma}=-\hat{J} \times \hat{z}, h$ is the reduced Planck constant, $e$ the elementary charge, $d_{\mathrm{FM}}$ 
the thickness of the FM layer, $\hat{J}$ the unit vector in the current direction, and $\hat{z}$ the unit vector along the $z$ axis; see Refs. [28,41]. However, such situations should not exist for a practical system, because many random factors, such as defects and thermal fluctuation, can disturb these ideal alignments. Once there is an $\mathbf{m}$ vector misaligned from $\pm \boldsymbol{\sigma}$, a finite SHT will arise and drag $\mathbf{m}$ toward $\boldsymbol{\sigma}$. Consequently, for any deviation from "m// $\mathbf{\sigma}$," the current-induced SHT will drive the system to return $\mathbf{m} / / \boldsymbol{\sigma}$; for any deviation from "m antiparallel to $\boldsymbol{\sigma}$ (i.e., $\mathbf{m} / /-\boldsymbol{\sigma})$," the SHT tends to drive $\mathbf{m}$ toward $\boldsymbol{\sigma}$. In the latter case, the rotation of $\mathbf{m}$ will destroy the given chirality of the SDWs that are defined by the IDMI. Further, the competition between the SHT and the torque associated with IDMI will give rise to chaotic SDW dynamics. Figure 4(c) illustrates the current-driven dynamics of an open strip domain without including the half-Skyrmion head [i.e., the part encircled by the box in Fig. 4(a)]. It is seen that one of the paired SDWs (here, the bottom SDW) begins to deform once the current is applied, and it is heavily distorted and intersects the bottom edge at 148 ps after the current application. Its subsequent chaotic motion will, in turn, destroy the other SDW (here, the top SDW) and result in disordered domain patterns in the nanowire. When the two SDWs are spatially separated [Figs. 4(d) and 4(e)], no interplay happens to them again. As a consequence, under the current action, one SDW sustains its initial geometry and always resides in the wire [Fig. 4(d)], while the other SDW distorts and finally breaks into pieces [Fig. 4(e)]. The results in Figs. 4(c)-4(e) serve as rigorous proof for the hypothesis stated above and illustrated in Figs. 3(g) and 3(h), and provide additional insight, along with Lin's latest theory [63], into the currentdriven dynamics of strip domains.

As shown in Fig. 4(b), after division, each SDW consists of a SDW ridge and a SDW head. The SDW head, which can be approximately regarded as a quarter-Skyrmion, has nonzero topological charge. Thus, under the current as displayed in Fig. 4(b), the bottom SDW cannot maintain its profile and will fluctuate irregularly, as how the SDW behaves in Fig. 3(f). However, under the same current, the top SDW can keep its general shape and gradually grow along the edge. This progressive elongation of the SDW benefits from the stabilization of the SDW ridge and the steady longitudinal motion of the SDW head along the edge. In fact, there is a transverse Magnus force acting on the quarter-Skyrmion-like SDW head as a result of its finite topological charge [62] (Appendix D, Fig. 14). Nevertheless, here, the SDW head is tied to the edge so that the confining force [64] coming from the edge cancels the Magnus force, and the transverse motion of the SDW head is suppressed completely, avoiding the zigzag distortion of the SDW and the creation of associated topological charge as described in Ref. [63]. That is to say, by splitting the strip domain with paired chiral SDWs into separate SDWs, each attached to an edge, the two independent physical mechanisms responsible for the destabilization of a strip domain can be simultaneously deactivated, and significantly, a single SDW can be imprinted into a wire by using the SHT.

In Ref. [32], strip domains were indeed observed to exist in the studied micrometer-sized samples subjected to ultralow current densities. However, the observed strip domains, which are deemed to be pinned and thereby extrinsically stabilized by the randomly distributed impurities inside the samples [63], exhibit an irregular zigzag profile, making the SDWs not suitable for guiding spin waves as smooth fibers. Furthermore, stabilization of the zigzag SDWs by impurities will become invalid for fast nanoscale devices that demand high-speed operation under reasonably high current densities.

\section{Influence of variations in geometry and material parameters}

We examine the applicability of the injection procedure with respect to variation in the samples' geometry, including the opening angle between the base and arms, the corner shape around the transition region, and the pad width of the Y-shaped nanostructure, and find that the injection procedure is generally valid throughout the considered geometries and also for the sample with vanishing IDMI. The details are presented in the Supplemental Material [27] Videos S1-S6.

The equilibrium domain patterns in the $90^{\circ} \mathrm{Y}$-shaped nanostructure relaxed from the as-written SDW for various values of $D$ (Fig. 5) clearly indicate that the IDMI strength must be in the proper range to stabilize the SDW at static equilibrium [Figs. 5(c)-5(e)]. For subthreshold $D$ values, the as-written SDW will transform into a multidomain texture [Fig. 5(a)] or it will disappear, resulting in a single domain [Fig. 5(b)]; for suprathreshold $D$ values, it will break into the labyrinthine wormlike texture shown in Fig. 5(f). The stability of SDWs in a Y-shaped nanostructure with respect to IDMI strength is different from the situation for a straight magnetic wire, where SDWs are stable even for $D=0$, as reported in Refs. [23,24]. This fact implies that the stabilization of a SDW in a bent wire requires a sufficiently strong IDMI. In the present study, the Y-shaped nanostructure includes a base, two arms, and a transition section, and the transition region between the base and the arm is a segment of a magnetic ring. Therefore, the dependence of SDW stability in a Y-shaped nanostructure on $D$ should be due to the presence of the transition region. For our Y-shaped nanostructures, the transition region is a 100-nm-wide arc with an outer radius of $400 \mathrm{~nm}$. We, thus, examine the stability of a SDW in such a $1 / 4$ arc against $M_{s}, K_{u}$, and $D$. The results displayed in Figs. 15-19 of Appendix G indicate that a SDW may be stabilized in the bent wire at static equilibrium over a broad range of values of $M_{s}, K_{u}$, and $D$.

\section{Current-controlled spin-wave multiplexers}

We now turn to the usefulness of the Y-shaped nanostructures, with controllably written SDWs, as spin-wave 


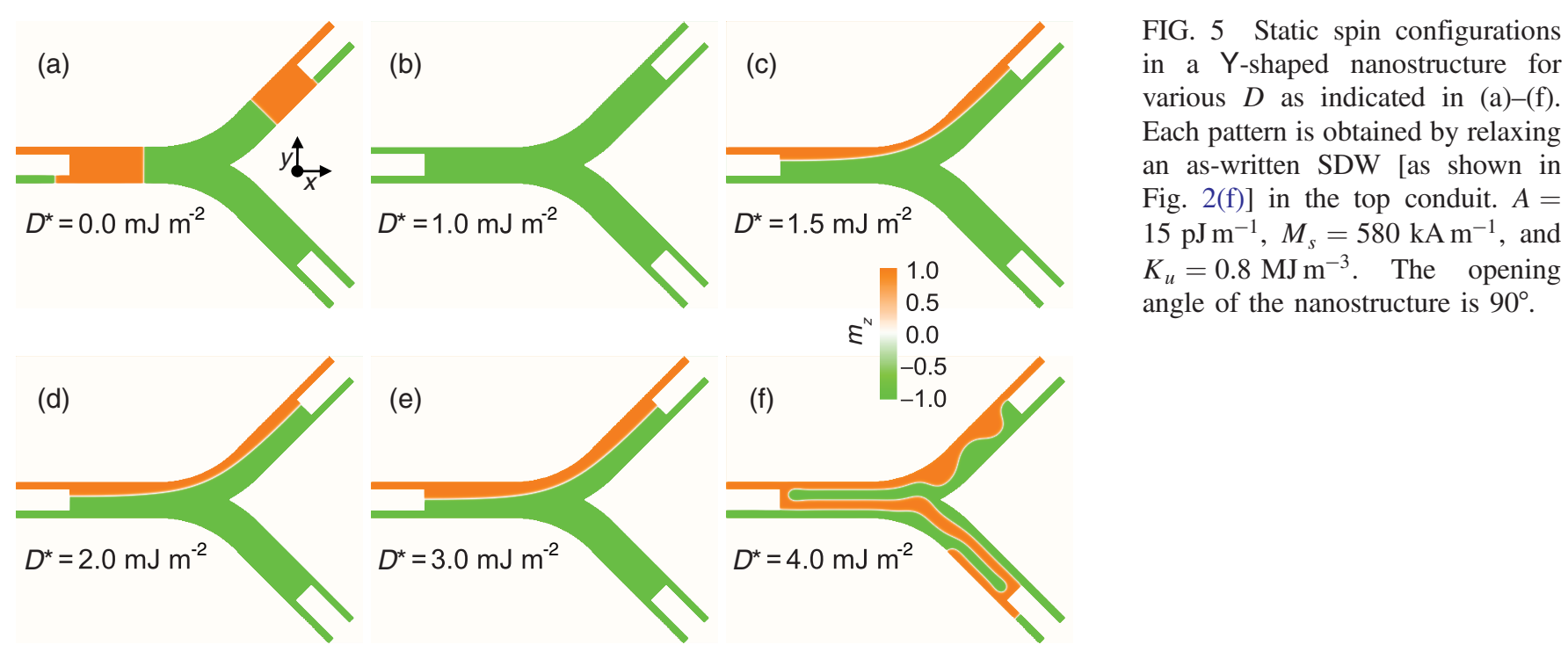

multiplexers. First, we examine spin-wave propagation in a $60^{\circ} \mathrm{Y}$-shaped nanostructure with a SDW placed in the top or bottom conduit or without a SDW. Figure 6 displays the propagation patterns of spin waves at $30 \mathrm{GHz}$. It can be seen that the spin waves are guided along a SDW in the top or the bottom conduit [Figs. 6(a) and 6(b)], but they do not exist inside the nanostructure without a SDW [Fig. 6(c)]. Here, regarding the channeling effect, the bottom arm in Fig. 6(a) and the top arm in Fig. 6(b) are equivalent to the corresponding arms in Fig. 6(c). Figures 6(d) and 6(e) plot the spin-wave amplitudes in symmetric zones of the $60^{\circ}$ nanostructure, with a SDW included in either arm, over a frequency range of $80 \mathrm{GHz}$, and suggest that the top and bottom SDWs are analogous as far as the channeling effect is concerned. The spin-wave strength in the arm with a SDW is more pronounced (approximately 1-2 times stronger) than that in the arm without a SDW. Note that a data point on the curves in Figs. 6(d) and 6(e) represents the spin-wave amplitude averaged over the area of the dashed box [Figs. 6(a) and 6(b)]; the actual difference between the spin-wave amplitude at the SDW position and that at the corresponding position without a SDW is, thus, underestimated. These results indicate that the $\mathrm{Y}$-shaped nanostructures can behave as current-controlled spin-wave multiplexers operating over a broad frequency band, which are expected to be of high energy efficiency owing to the
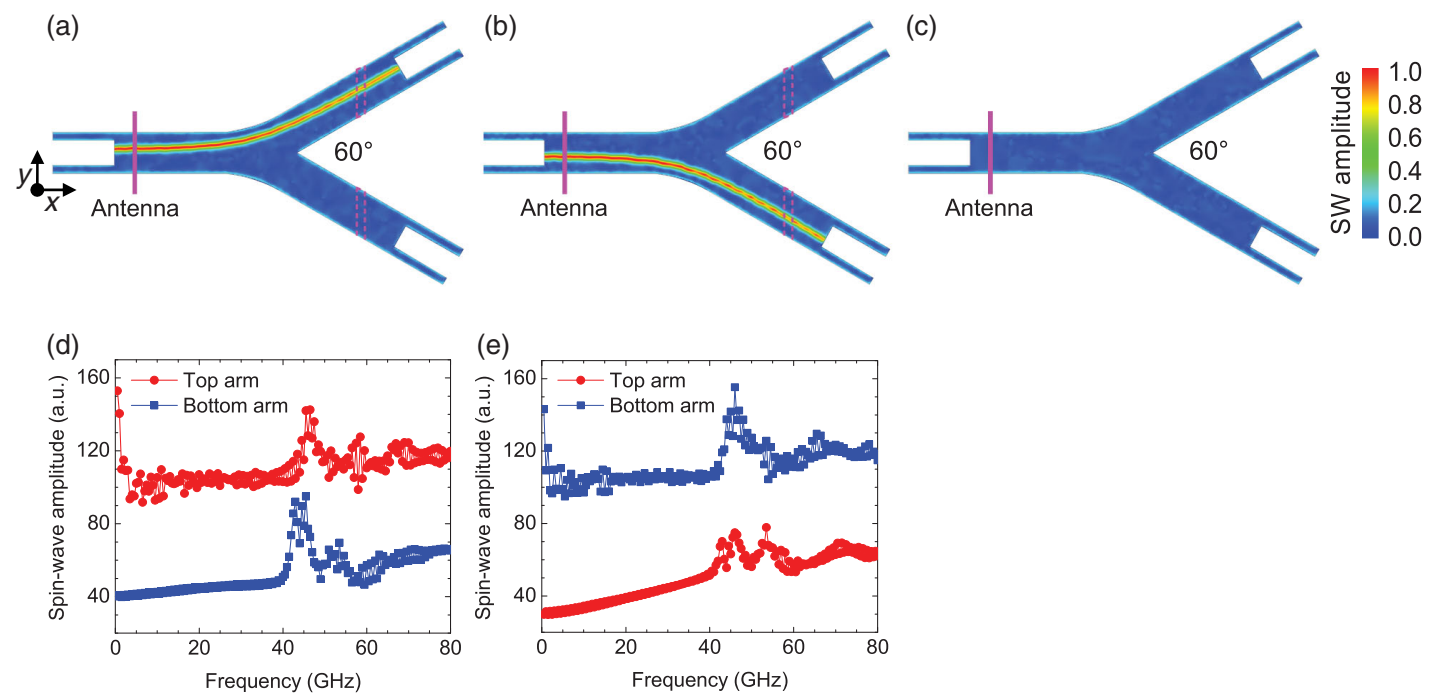

FIG. 6 Y-shaped nanostructure with controllably written SDW as the spin-wave multiplexer. (a) Spin-wave transmission along the top conduit with a SDW. (b) Spin-wave transmission along the bottom conduit with a SDW. (c) Spin-wave transmission prohibited in the nanostructure without including SDWs. (d), (e) Comparison of spin-wave amplitudes on the two arms with various spin configurations. Plots (d) and (e) correspond to plots (a) and (b), respectively. Spin waves are excited at the antenna. The spin-wave frequency is 30 GHz. The opening angle of the $\mathrm{Y}$-shaped nanostructure is $60^{\circ}$. 


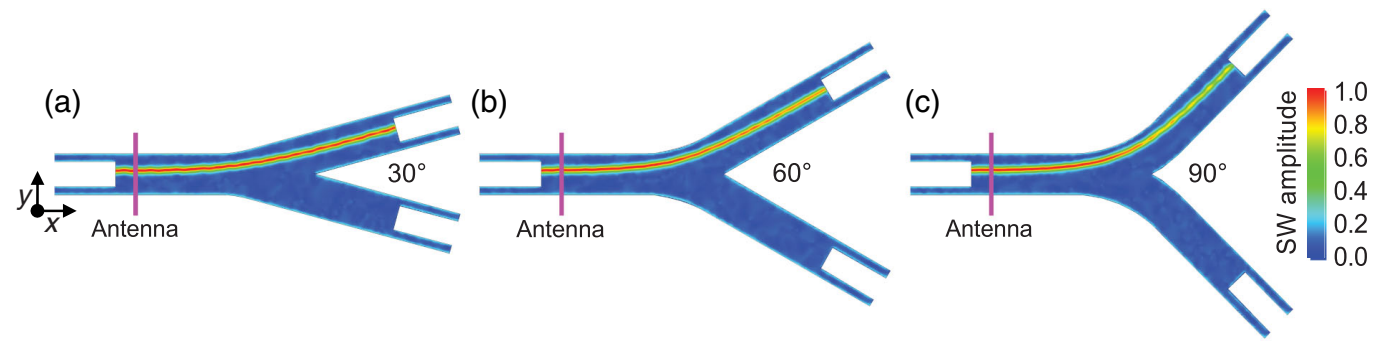

FIG. 7 Spin-wave transmission in a multiplexer dependent on opening angles. The opening angles for the multiplexers in (a), (b), and (c) are $30^{\circ}, 60^{\circ}$, and $90^{\circ}$, respectively. Spin waves are excited at the antenna. The spin-wave frequency is $30 \mathrm{GHz}$.

nonvolatility of the rewritable spin-wave guiding channels. Such low power consumption, as a key figure of merit for device applications [65], is difficult to achieve in the multiplexer based on Damon-Eshbach spin-wave channels maintained by the current-induced Oersted field recently proposed in Ref. [11].

In a further step, spin-wave propagation along SDWs inside the $\mathrm{Y}$-shaped nanostructures with different opening angles is examined; the results are shown in Fig. 7. Clearly, for all three opening angles, the SDWs in the Y-shaped nanostructures can channel spin waves, indicating that the SDW-based spin-wave multiplexer can operate over a wide angle range. Although for all the three opening angles, the spin waves can travel smoothly along the SDWs, the spin waves for larger opening angles decay faster after passing through the corner, consistent with Ref. [11]. That is to say, the larger the opening angle is, the heavier the spin-wave attenuation with the propagation distance; this is possibly because the spin waves in multiplexers with larger opening angles experience stronger intermodal scattering or boundary scattering [21,66-70].

To excite spin waves, we adopt the inductive method based on a strip-line antenna, which produces a magnetic field with a symmetric profile relative to the SDW elongation axis. With this inductive scheme, only spin waves with $2 n(n=1,2,3 \ldots)$ nodes can be activated, and the excitation efficiency of a mode is proportional to $1 /(2 n-1)^{2}$, where $2 n-1$ is the order of the excited mode $[70,71]$. Consequently, the ratio between the nominal excitation strengths of the third-order and first-order modes is $1 / 9$. In particular, only the fundamental mode is strongly excited at the antenna. When the fundamental mode runs into the bent section of a SDW, the $2 n$-order mode can be activated because of the translation-symmetry breaking $[67,70]$. In this way, the fundamental mode can scatter into higher-order modes [72]. An increased opening angle
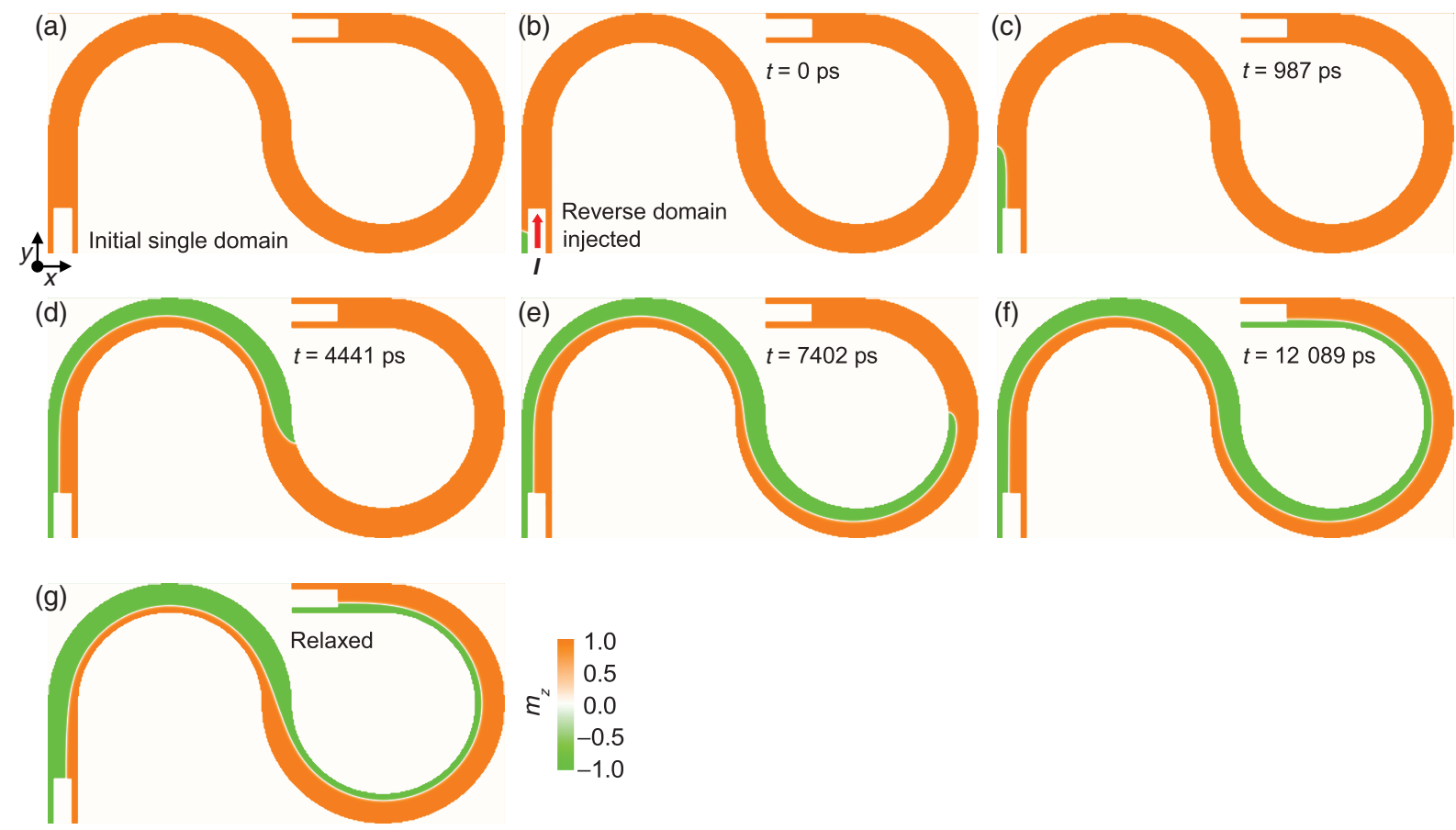

FIG. 8 SDW injection into an S-shaped nanowire. (a) Initial single-domain state. (b) Seed domain formed at a nucleation pad. Current is switched on at 0 ps when the seed domain wall just forms. (c)-(f) Transient-state SDWs at indicated times after current application. (g) Static SDW after relaxation from 12089 ps. 
makes a transmission channel undergo stronger bending, which, in turn, leads to enhanced intermodal scattering and, thus, enhanced attenuation of the fundamental mode.

On the other hand, the distance between the SDW and the top edge decreases as the opening angle increases, as seen in Fig. 7. This reduced separation has two effects: first, the fiber mode (the mode confined in the SDW) [23] will mix with the edge mode $[66,73,74]$ because of the overlap of their spatial profiles; second, the fiber mode will be scattered by the boundary defects [66], such as edge roughness. The modal scattering between the fiber and edge modes, as well as boundary scattering, become two additional energy-dissipation channels for the fundamental fiber mode.

\section{E. Writing a strip-domain wall into an S-shaped wire}

To ensure that the proposed SDW-injection procedure is generally valid for bent wires, we use it to write a SDW into an S-shaped wire, whose bent parts are 100-nm-wide arcs with an outer radius of $400 \mathrm{~nm}$. The whole process resembling that in Fig. 2 is illustrated in Fig. 8. The initial state is a single domain with $m_{z}=+1$. A seed reverse domain is formed in a nucleation pad by using a magnetic field pulse, and then an electric current is fed into the HM2 layer of the wire from the lower-left to the upper-right terminal. The 0-ps point in Fig. 8(b) marks the time when the electric current is switched on. Once the current is applied, the seed domain wall moves into the wire and becomes a SDW [Fig. 8(c)]. Under the continuous action of the current, the SDW moves forward and ultimately extends over the entire length of the wire [Figs. 8(d)-8(f)]. A variation in strip-domain width with position [Fig. 8(g)] also occurs in the SDWs in the Y-shaped nanostructures [Figs. 2(f) and $2(\mathrm{~g})$ ], which can be ascribed to the compromise between the various competing energy terms.

A micromagnetic system tends to reach an energy minimum by releasing extra energy. When a straight wire is bent, inclusion of the concaves and convexes will modify the energy landscape of the entire system, and the SDW will adapt to the curvature by varying the strip-domain width to reach a new energy minimum. The strip domain with a varying width enables the whole system to stay at a (meta)stable equilibrium state with lower energy.

\section{DISCUSSION}

For the systems considered here, Néel domain walls are the preferred type for rendering the most efficient domain-wall motion by SHT, as found in Refs. [30,46]. During the SDW-injection process (Fig. 2), the effective field $\left(\mathbf{H}_{\mathrm{SH}} \propto \mathbf{m} \times \boldsymbol{\sigma}\right.$ [46]) associated with SHT exerting on the magnetization at the SDW head is aligned with the magnetization of the newly formed reverse domain, and, thus, the SDW head moves forward through the continuous expansion of the reverse domain along an edge of the wire. However, the effective field in the SDW ridge vanishes because of the parallel alignment of the electron spins and the magnetization in the SDW ridge [Fig. $3(\mathrm{~g})]$ so that after formation, the SDW ridge does not experience a torque and, thus, stops.

The Rashba effect has been suggested as the main driving force for domain-wall motion in a $\mathrm{Pt} / \mathrm{Co} / \mathrm{AlO}_{x}$ wire [75]. It is, thus, essential to identify whether the Rashba effect plays a key role in the present case. But, as we see from simulations, the Rashba torque $\left[\mathbf{T}_{f}=\right.$ $-\gamma \tau_{R}(\mathbf{m} \times \boldsymbol{\sigma})$, where $\tau_{R}=J P \alpha_{R} / \mu_{B} \mu_{0} M_{s}$ and $\mu_{B}$ is the Bohr magneton; see Ref. [47]] coexisting with SHT does not significantly change the SDW-injection process as long as the relative strength of RT to SHT $\tau_{R} / \tau_{H}$ is not higher than 0.5 (see Appendix A for details). We pass the electric current only to the HM2 layer, rather than to the entire thickness by using $\mathrm{AO}_{x}$ as an isolation element to avoid the conventional Zhang-Li spin torques and, thus, ensuring that only the SOT exists in the FM layer. By doing so, the computations can be greatly simplified, since the relative strength of the nonadiabatic and adiabatic torques is not definitely known. Experimentally, in principle, eliminating the Zhang-Li torques is not required for SDW injection in the $\mathrm{HM} 1 / \mathrm{FM} / \mathrm{AO}_{x} / \mathrm{HM} 2$ system because the Zhang-Li torque accompanying SHT alters only the effective velocity of the SDW head (Appendix A, Fig. 10), contributing negligibly to domain-wall displacement in ultrathin multilayer nanostructures, as argued in Ref. [30]. Notably, the proposed procedure can work at room temperature and is not sensitive to impurities inside the sample even for an ultrahigh impurity concentration of 5\% (Appendix B, Fig. 12).

Different from the approach presented in Ref. [23], which seems to apply only to straight magnetic wires, the method proposed here can work well for SDW injection in long magnetic wires with curvature (even at room temperature, as shown in the Supplemental Material [27] Video S7). On the other hand, the method used in Ref. [23] requires a spin valve or magnetic tunnel junction to produce a spin-polarized current, so the device structure is complex. By contrast, the present writing scheme does not require excessive units to generate a spin current. Another difference between the two injection schemes is that the former method is based on magnetization switching to form a reverse domain, whereas the present method relies on the motion of a domain-wall head to yield a reverse domain. Once the reverse domain forms in the background domain, the injection of a SDW situated between the initial and reverse domains is achieved. From the viewpoint of applications, bent components $[21,67,68,70,76]$ will be an unavoidable building block in functional magnonic circuits $[7,10,11]$. The present method is well suited for writing SDWs into realistic circuits with bent parts to form fiber-type magnonic waveguides [22-24]. 
The drawback of the present SDW-injection method is that it is invalid for all domain-wall types other than Néel walls. For example, it is not compatible with Bloch or transverse walls. It can, thus, be used only in material systems with PMA and IDMI that favor the Néel-type domain wall in a magnetic wire [77]. The recently reported ultralow magnetic damping of approximately $10^{-4}$ of sputter-deposited polycrystalline $\mathrm{CoFe}$ films with $\mathrm{Cu} / \mathrm{Ta}$ seed and capping layers [78], previously attained only for ferrimagnetic YIG films [79,80], can exhibit all the required PMA, IDMI, and SOT [30] and, therefore, might bring an unprecedented opportunity for the demonstrated SDW-injection procedure [81] and the established spinwave guiding scheme based on SDWs. However, the commonly used material systems for present-day magnonic applications are low-damping Py [1,3] and YIG [82] films without PMA, for which the proposed injection scheme will fail because of mismatching between domain-wall configuration and SOT [46].

Although the structure of our multiplexers is similar to that in Ref. [11] and we also use an electric current to set a spin-wave channel, our waveguide mechanism [22-24] is different; in particular, no current is needed to maintain the spin-wave channels in our multiplexers after imprinting, leading to substantially reduced power consumption. Additionally, the energy benefit and its applicability to bent samples might make our waveguide outperform the one exploited in Refs. [19,20].

\section{CONCLUSION}

We propose a robust method for writing strip-domain walls into magnetic nanostructures patterned from an ultrathin multilayer film with perpendicular magnetic anisotropy, the interfacial Dzyaloshinskii-Moriya interaction, and the spin Hall effect, and even containing highconcentration impurities. Apart from straight wires, the method can be used for bent samples even at room temperature. Moreover, the spin-wave waveguide and multiplexer based on strip-domain walls are energy efficient compared to previously established ones. These findings may drive them to become prototypical spin-wave devices in magnonics. We also identify an emergent physical mechanism for the stabilization or destabilization of a domain wall under the spin Hall torque, which might lead to operation concepts in domain-wall-based memory and logic devices.

\section{ACKNOWLEDGMENTS}

Y.Z. acknowledges the support by the National Natural Science Foundation of China (Project No. 11574137) and the Shenzhen Fundamental Research Fund under Grant No. JCYJ20160331164412545. X. J. X. acknowledges the support of the National Natural Science Foundation of China under Grants No. 11104206 and No. 61675050.

\section{APPENDIX A: EFFECT OF RASHBA TORQUE AND ZHANG-LI TORQUE ON STRIP-DOMAIN-WALL INJECTION}

We examine how the RT and STT affect the injection process of a SDW driven by the SHT. For the RT, a set of Rashba parameters $\alpha_{R}$ ranging from $-1.5 \times 10^{-21}$ to $1.5 \times 10^{-21} \mathrm{erg} \mathrm{cm}$ are considered with an interval of $0.1 \times 10^{-21} \mathrm{erg} \mathrm{cm}$. For STT, we consider that $\beta=\alpha$, and we adjust the current direction and the sign of the

(a)

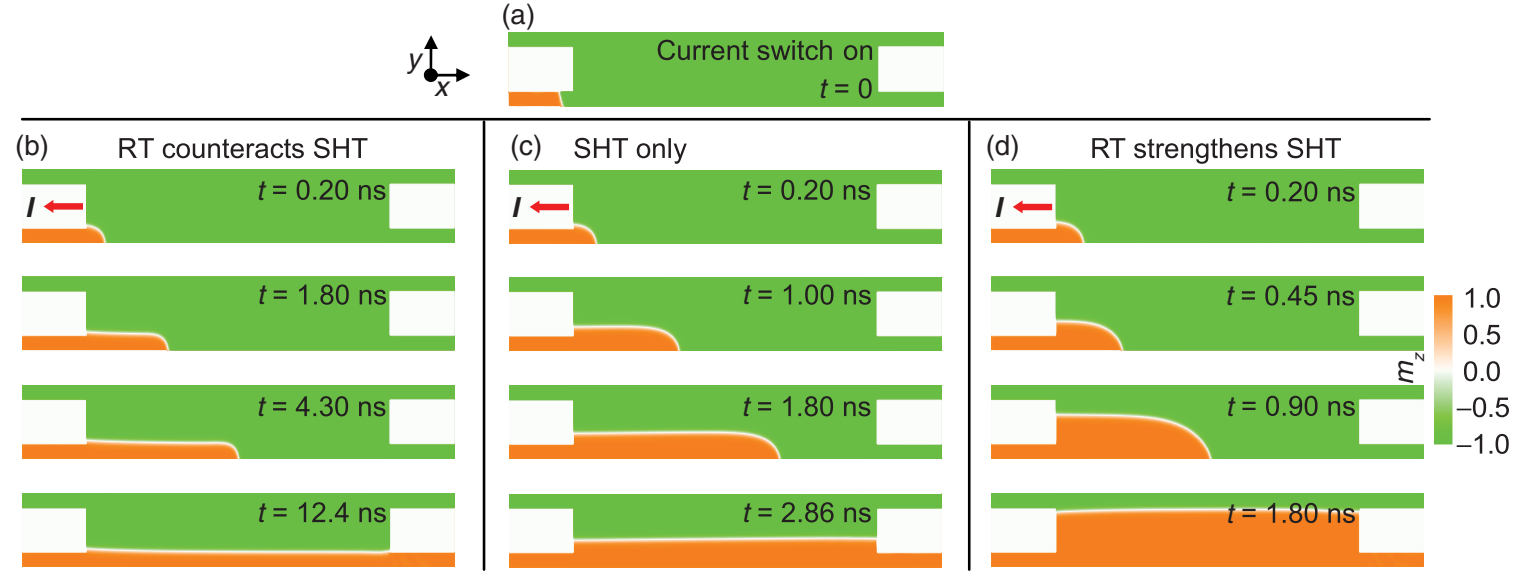

FIG. 9 Effect of Rashba torque coexisting with the spin Hall torque on SDW injection. Here, $D=-2 \mathrm{~mJ} \mathrm{~m}^{-2}, \Phi_{H}=+0.13, P=0.4$, and we assume that $J_{\mathrm{FM}}=J_{\mathrm{HM} 2}=3 \times 10^{12} \mathrm{Am}^{-2}$. (a) Seed reverse domain nucleated at the lower-left pad. Current is turned on at the time $t=0 \mathrm{~ns}$ when the seed domain is just formed. Snapshots of the SDW at various times after the current action for (b) $\alpha_{R}=+0.5 \times 10^{-21} \mathrm{erg} \mathrm{cm}$, (c) $\alpha_{R}=0$, and (d) $\alpha_{R}=-0.5 \times 10^{-21} \mathrm{erg} \mathrm{cm}$. Note that for $\alpha_{R}= \pm 0.5 \times 10^{-21}$ erg cm, the relative magnitude of RT to SHT is $\left|\tau_{R} / \tau_{H}\right|=0.5$. The Rashba torque induces transverse shift of the SDW ridge and suppresses (strengthens) the drift motion of the SDW head along the edge for a positive (negative) Rashba parameter $\alpha_{R}$. 
(a)

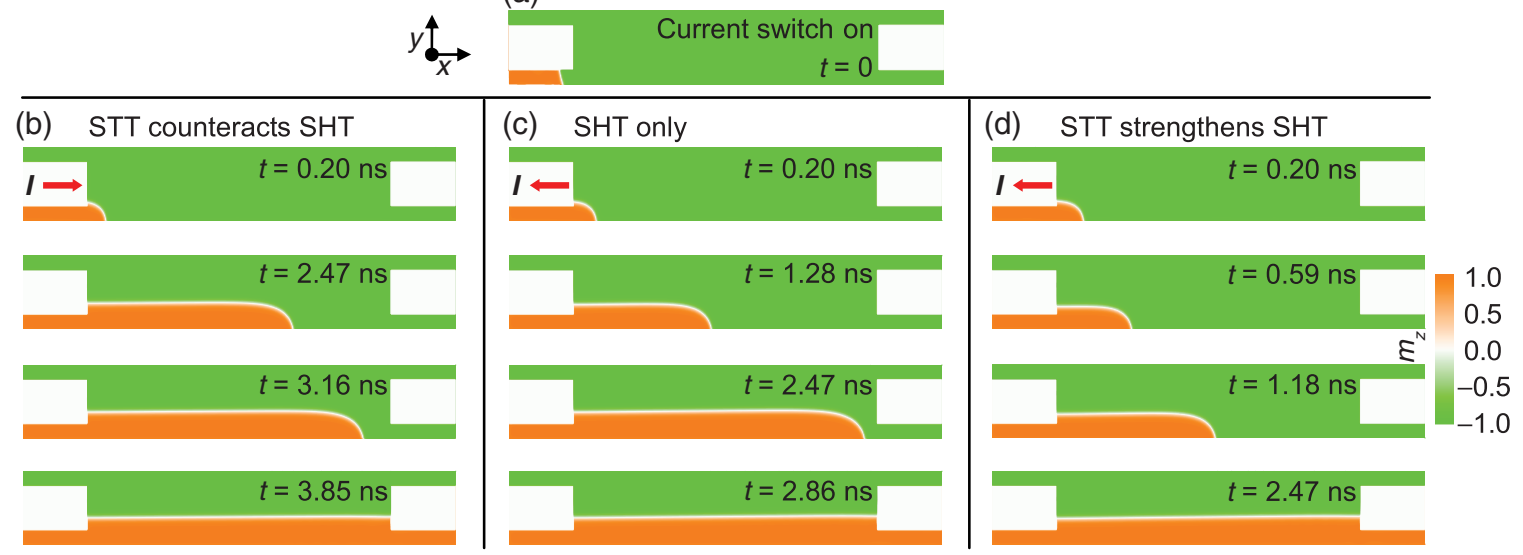

FIG. 10. Effect of Zhang-Li spin-transfer torque coexisting with the spin Hall torque on SDW injection. Here, $D=-2 \mathrm{~mJ} \mathrm{~m}^{-2}$, $P=0.4$, and we assume $\beta=\alpha$. (a) Seed reverse domain nucleated at the lower-left pad. Current is turned on at the time $t=0 \mathrm{~ns}$ when the seed domain is just formed. Snapshots of the SDW at various times after the current action for (b) $\Phi_{H}=-0.13$ and $J_{\mathrm{FM}}=$ $J_{\mathrm{HM} 2}=3 \times 10^{12} \mathrm{Am}^{-2}$ along $+x$, (c) $\Phi_{H}=+0.13, J_{\mathrm{FM}}=0$, and $J_{\mathrm{HM} 2}=3 \times 10^{12} \mathrm{Am}^{-2}$ along $-x$, and (d) $\Phi_{H}=+0.13$ and $J_{\mathrm{FM}}=$ $J_{\mathrm{HM} 2}=3 \times 10^{12} \mathrm{Am}^{-2}$ along $-x$. Note that STT can suppress or enhance SHT, depending on the direction of applied current and the spin Hall angle.

spin Hall angle $\Phi_{H}$ to make the STT counteract or strengthen the SHT. We find that the SDW-injection process is not significantly affected by the RT or STT coexisting with the SHT under the condition $J_{\mathrm{FM}}=J_{\mathrm{HM} 2}$ if the relative strength of the RT to SHT is not higher than 0.5. The details are presented in Figs. 9 and 10. We also compare the dynamics of the SDW and conventional domain wall; the results are shown in Fig. 11.

In Fig. 11(a), below $J_{c}$, the SHT-related force cannot overcome the elastic restoring force, and the SDW head in the nanowire does not move. At $J_{c}$, the elastic restoring force is just balanced by the SHT-related force, and the (a)

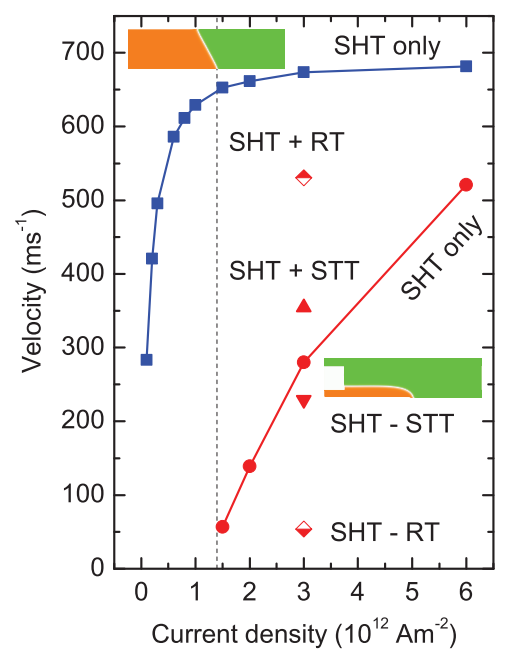

(b)

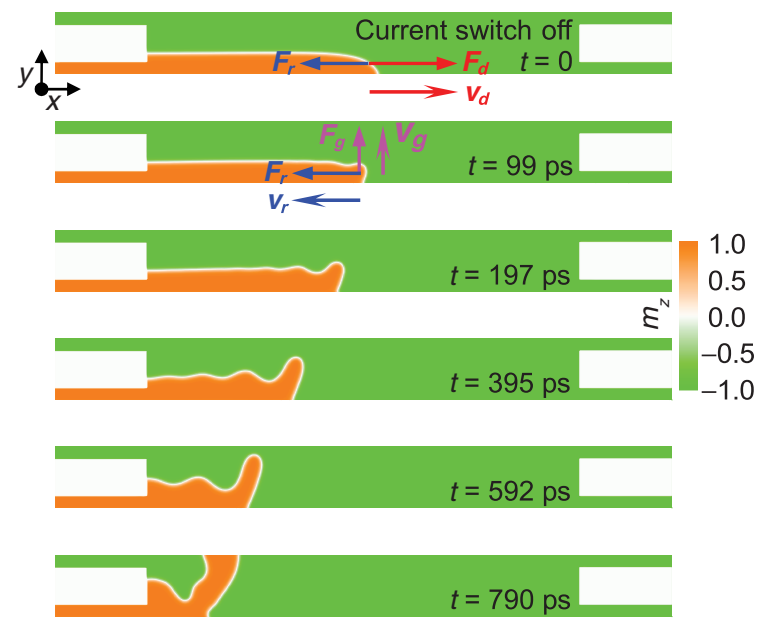

FIG. 11. (a) Domain-wall velocity and SDW-head velocity versus current density. The blue squares represent the velocity values of the domain wall subjected to SHT only. The red circles denote the velocity values of the SDW subjected to SHT only. The dashed vertical line labels the critical current density $J_{c} \approx 1.35 \times 10^{12} \mathrm{Am}^{-2}$, above which the SDW head in the nanowire can be moved by SHT. The triangles give the velocity values of the SDW under coexisting SHT and STT. "SHT+STT" ("SHT-STT") indicates that STT enhances (suppresses) the SDW motion under SHT. The diamonds stand for the velocity values of the SDW under coexisting SHT and RT. "SHT + RT" ("SHT-RT") indicates that RT enhances (suppresses) the SDW motion under SHT. Inserted into the graph are the domain wall (top) and SDW (bottom). $J_{\mathrm{FM}}=J_{\mathrm{HM} 2}$ is assumed. (b) Relaxation dynamics of a SDW. The current $J_{\mathrm{HM} 2}=3 \times 10^{12}$ Am ${ }^{-2}$ suddenly stops at the time $t=0 \mathrm{ps}$ when the SDW head is halfway to the right end of the nanowire. All data in (a) and (b) are simulated with $D=-2 \mathrm{~mJ} \mathrm{~m}^{-2}$. 


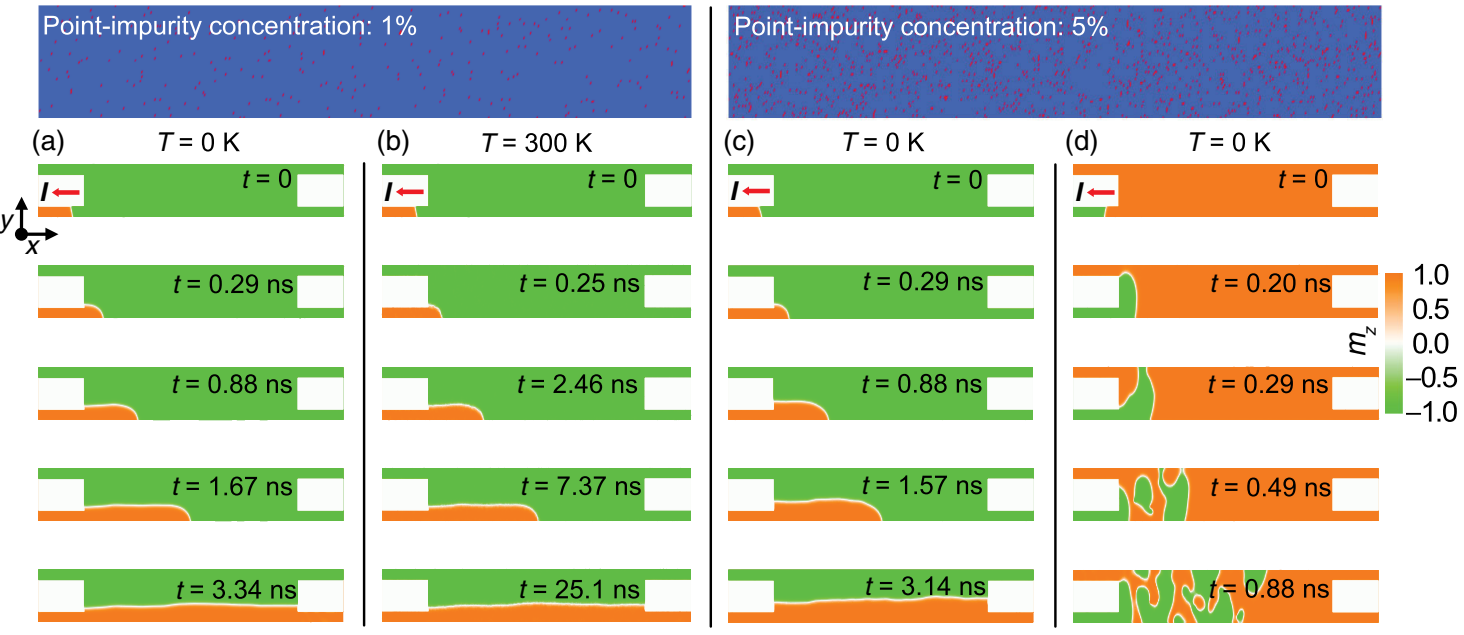

FIG. 12. Effect of impurities on the SDW injection driven by SHT. The top-left and top-right panels display the impurity distributions for the impurity concentration $c=1 \%$ and $5 \%$, respectively. $D=-2 \mathrm{~mJ} \mathrm{~m}^{-2}$ for the nonimpurity sites, whereas $D=2 \mathrm{~mJ} \mathrm{~m}^{-2}$ for the impurity sites. $J_{\mathrm{HM} 2}=3 \times 10^{12} \mathrm{Am}^{-2}$. SDW-injection process for the $c=1 \%$ nanowire at the temperature (a) $T=0 \mathrm{~K}$ and (b) $T=300 \mathrm{~K}$. SDW-injection process for the $c=5 \%$ nanowire at $T=0 \mathrm{~K}$ initiated from (c) a right seed domain and (d) a wrong seed domain.

SDW head will be kept stationary. Above $J_{c}$, the SDW head in the nanowire can be displaced by the SHT. Note that $J_{c}$ does not apply to the cases where the RT or STT accompanies the SHT. As seen from Fig. 11(a), the critical current density for the SDW is much higher than that for the conventional domain wall, which should be attributed to the higher elastic energy of the former, as revealed in Fig. 11(b). We do not see auto-oscillation in SDWs in the covered current-density range up to $6 \times 10^{12} \mathrm{Am}^{-2}$ [83]. In Fig. 11(b), before the current stops, the current-induced force $F_{d}$ on the SDW head is balanced by the restoring force $F_{r}$, and the SDW head moves steadily at a constant speed $v_{d}$. Once the current is removed, $F_{d}$ disappears, and the SDW head contracts rapidly at a negative speed $v_{r}$ under the elastic restoring force originating from the high elastic energy of the SDW. The speed $v_{r}$ causes a transverse Magnus force on the SDW head because of the finite topological charge of the latter. In turn, the Magnus force drags the SDW head upward (i.e., along $+y$ ), and eventually, the ordered SDW structure is destroyed. This simulated dynamic process is consistent with Thiele's theory [84].

\section{APPENDIX B: EFFECT OF POINT IMPURITIES ON STRIP-DOMAIN-WALL INJECTION}

The nanowires modeled are assumed to be patterned from ultrathin multilayer films, and, thus, defects can never be totally avoided in real samples. Defects were reported to heavily influence the motion of Skyrmions driven by a current [33]. This fact stimulates us to consider the effect of defects on the SDW injection driven by SHT. We consider point impurities randomly distributed inside the nanowire and find that even for an ultrahigh concentration of impurities, 5\%, the SDW injection can still be realized satisfactorily. The details are presented in Fig. 12. Because of the pinning effect of impurities, the SDWs imprinted into the nanowires containing impurities are not as smooth as that in a perfect wire.

Comparing Figs. 2, 3, and 12 and Video S7 in the Supplemental Material [27], it is clear that a SDW can be successfully written into a nanowire if a correctly set seed domain is used, irrespective of the geometry of the nanowire (straight or bent), the defect concentration in the wire (with or without including impurities), and the operating temperature $(0$ or $300 \mathrm{~K})$.

\section{APPENDIX C: FAILURE TO INJECT A STRIP DOMAIN WITH PAIRED CHIRAL STRIP-DOMAIN WALLS}

In principle, a strip domain cannot be written into a nanowire by using SHT, because, on one hand, the halfSkyrmion attached to the end of the strip domain will distort the SDW (a mechanism proposed recently in Ref. [63] by Lin), and, on the other hand, one of the paired SDWs of a strip domain will be directly destabilized by SHT (an independent mechanism proposed in the present paper; see Fig. 4). Although we are aware of this fact, we still attempt to inject a strip domain with paired SDWs into a wire. However, as expected, no strip domains are injected into the wire, and instead, a SDW is written into the wire. The details are presented in Fig. 13. Obviously, only the SDW in which $\mathbf{m} / / \boldsymbol{\sigma}$ is permitted to reside in the nanowire, and the other SDW, in which $\mathbf{m}$ is antiparallel to $\boldsymbol{\sigma}$, is always expelled from the nanowire. Consequently, the desired strip domain with paired SDWs can never form, regardless of the orientation of the seed domain. 
(a)
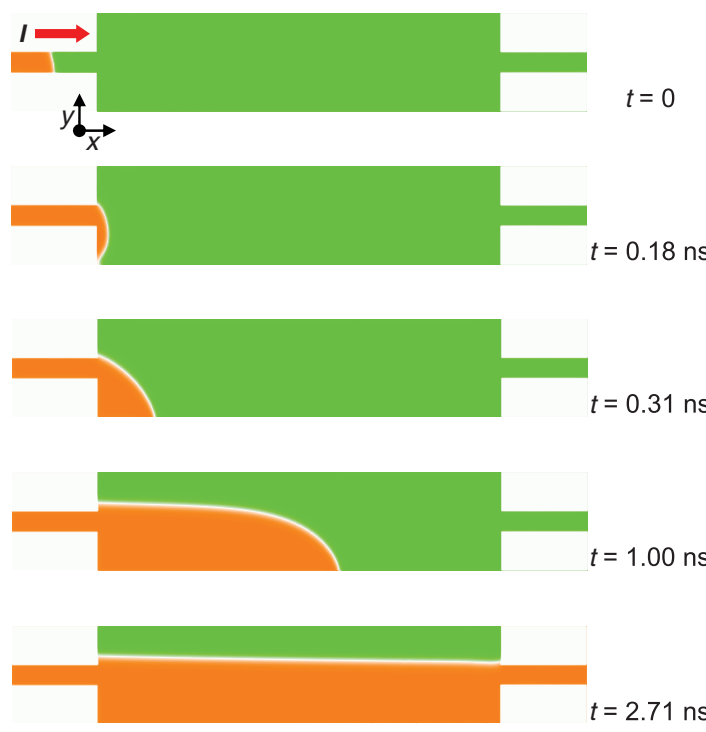

(b)
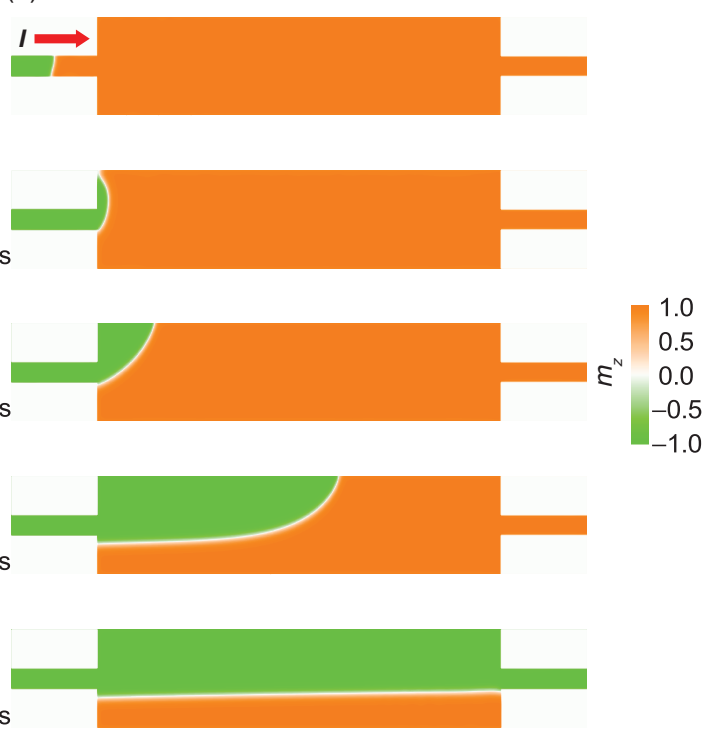

FIG. 13. Failure to inject a strip domain with paired chiral SDWs. Here, $D=2 \mathrm{~mJ} \mathrm{~m}^{-2}$ and $J_{\mathrm{HM} 2}=3 \times 10^{12} \mathrm{Am}^{-2}$. The nucleation pads are placed along the central axis of the sample to maintain the mirror symmetry. The seed domains in (a) and (b) are upward and downward, respectively. From $t=0$ on, the current is applied. In each case, a SDW instead of a strip domain, with paired SDWs, is imprinted into the wire.

\section{APPENDIX D: DYNAMICS OF A FRACTIONAL SKYRMION}

A closed strip domain always has two half-Skyrmion heads, each of which is tied to an end of the strip domain, whereas a SDW also includes a head similar to a quarterSkyrmion. Both the half-Skyrmion and quarter-Skyrmion carry finite topological charge and, thus, are expected to show topological dynamic behavior. Here, the halfSkyrmion (quarter-Skyrmion) head is bound to the linear part of the entire structure so that it is impossible to clearly see its topological behavior. We, therefore, study the current-induced dynamics of a meron (i.e., fractional Skyrmion) to identify the forces experienced by the SDW head and understand its behavior. The details are presented in Fig. 14, where these current-induced forces experienced by the standalone meron should also occur in the half-Skyrmion attached to the strip domain [Fig. 4(a)] and occur in the quarter-Skyrmion head bound to the SDW (a)

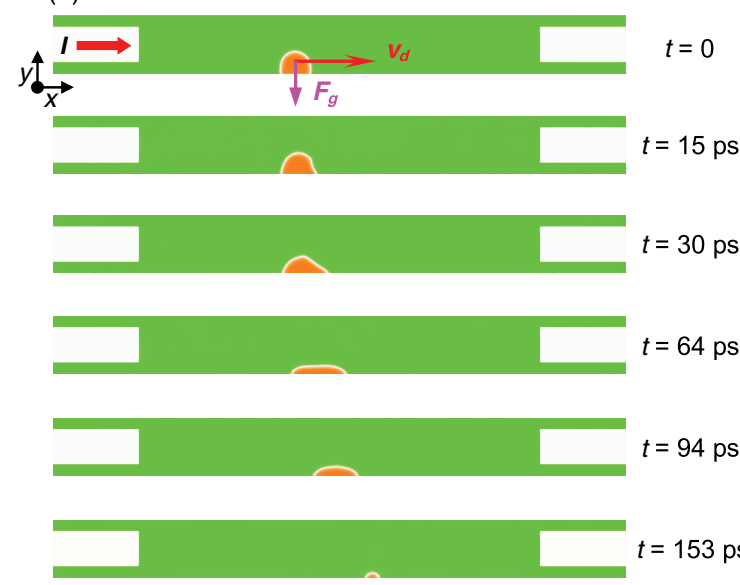

(b)
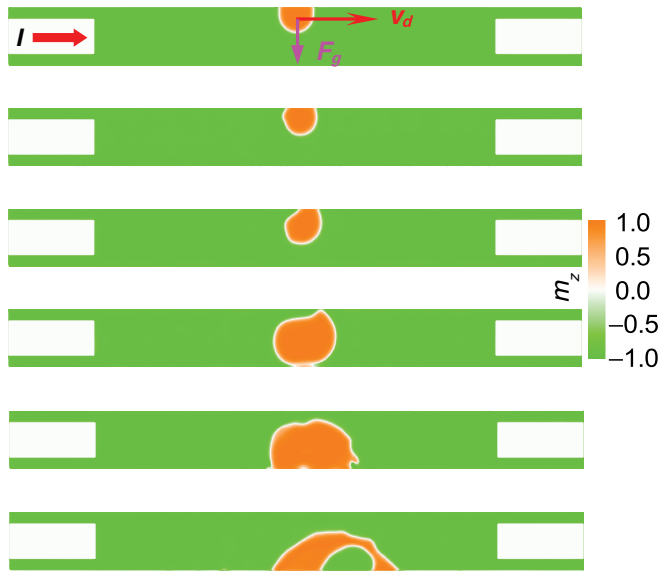

FIG. 14. Dynamics of a meron, a magnetic object with fractional topological charge. Here, $D=3.5 \mathrm{~mJ} \mathrm{~m}^{-2}$ and $J_{\mathrm{HM} 2}=3 \times 10^{12} \mathrm{Am}^{-2}$. Initially, the meron sits at (a) the bottom edge and (b) the top edge of the nanowire. Under the current, the meron moves along the edge and, thus, acquires a longitudinal speed $v_{d}$. The longitudinal motion then causes a Magnus force $F_{g}$ to act on the meron owing to the meron's nonzero topological charge. The Magnus force expels the meron from the nanowire in (a) and drags the meron into the nanowire in (b). 
[Fig. 4(b)]. Therefore, the SDW head in the top panel of Fig. 4(b) feels an outward force (i.e., along $-y$ ), which stabilizes the SDW, whereas the SDW head in the bottom panel of Fig. 4(b) undergoes an inward force (i.e., along $-y$ ), which distorts the SDW according to Lin's theory [63].

\section{APPENDIX E: VALIDITY OF THE INJECTION PROCEDURE AGAINST GEOMETRIC VARIATION}

The injection procedure is valid for $\mathrm{Y}$-shaped nanostructures with $60^{\circ}$ and $30^{\circ}$ opening angles, as shown in the Supplemental Material [27] Videos S2 and S3 [27], respectively. Apart from the pad width of $w_{N}=20 \mathrm{~nm}$, other width values of $w_{N}=30$ and $40 \mathrm{~nm}$ are examined, as shown in Videos S4(a) and S4(b) in Ref. [27]. We find that the larger the pad width, the wider the imprinted strip domain [compare Videos S1(a), S4(a), and S4(b) in Ref. [27] ]; however, following relaxation, the static SDWs are identical. The injection process is tested against a sharp corner around the transition region of the $90^{\circ} \mathrm{Y}$ shaped nanostructure (Video S5 in Ref. [27]), and it can be seen that the SDW head can pass through the sharp corner.

\section{APPENDIX F: VALIDITY OF THE INJECTION PROCEDURE UNDER VANISHING DZYALOSHINSKII-MORIYA INTERACTION}

Significantly, this injection procedure can also be applied to samples without IDMI, as shown in Video S6 in Ref. [27] ]. Comparing Video S1(a) $\left(D=2.0 \mathrm{~mJ} \mathrm{~m}^{-2}\right)$ with Video S6 $(D=0)$ in the Supplemental Material [27], it can be seen that there is no difference between the injection processes, which indicates that the injection procedure does not rely on the presence of IDMI. Nevertheless, SDWs cannot be stabilized in the $\mathrm{Y}$-shaped nanostructure with $D=0$; once the writing current is removed, the written SDW deforms rapidly and collapses, resulting in an undesired equilibrium domain pattern, as shown in Fig. 5(a). This fact highlights the importance of identifying the parameter space where a SDW can exist at static equilibrium.

\section{APPENDIX G: DEPENDENCE OF STATIC DOMAIN STATE ON MATERIAL AND GEOMETRIC PARAMETERS}

The equilibrium domain patterns in the $90^{\circ} \mathrm{Y}$-shaped nanostructure relaxed from the as-written SDW for various values of $D$ (Fig. 5), clearly indicate that the IDMI strength must be in a proper range to stabilize the SDW at static equilibrium [Figs. 5(c)-5(e)]. For subthreshold $D$ values, the as-written SDW will transform into a multidomain texture [Fig. 5(a)] or will disappear, resulting in a single domain [Fig. 5(b)]; for suprathreshold $D$ values, it will break into the labyrinthine wormlike texture shown in Fig. 5(f). The stability of SDWs in a Y-shaped nanostructure with respect to IDMI strength is different from the situation for a straight magnetic wire, where SDWs are stable even for $D=0$, as reported in Refs. [23,24]. This fact implies that the stabilization of a SDW in a bent wire requires a sufficiently strong IDMI. In the present study, the Y-shaped nanostructure includes a base, two arms, and a transition section, and the transition region between the base and the arm is a segment of a magnetic ring. Therefore, the dependence of SDW stability in a Y-shaped nanostructure on $D$ should be due to the presence of the transition region.

For our Y-shaped nanostructures, the transition region is a 100-nm-wide arc with an outer radius of $400 \mathrm{~nm}$. We, thus, examine the stability of a SDW in such a $1 / 4$ arc against $M_{s}, K_{u}$, and $D$; the results are displayed in Figs. $15-17$, indicating that a SDW may be stabilized in the 1/4-arc wire at static equilibrium over a broad range of values of $M_{s}, K_{u}$, and $D$. As expected, the arc has a similar SDW-stability range in $D$ to the $\mathrm{Y}$-shaped nanostructure (compare Figs. 5 and [17]). To clarify the influence of curvature, we check the SDW stability against $D$ in a set of arcs with the same width $(100 \mathrm{~nm})$ but different radii (400, 800, and $1200 \mathrm{~nm}$ ), as shown in Fig. 20(a). We find that the

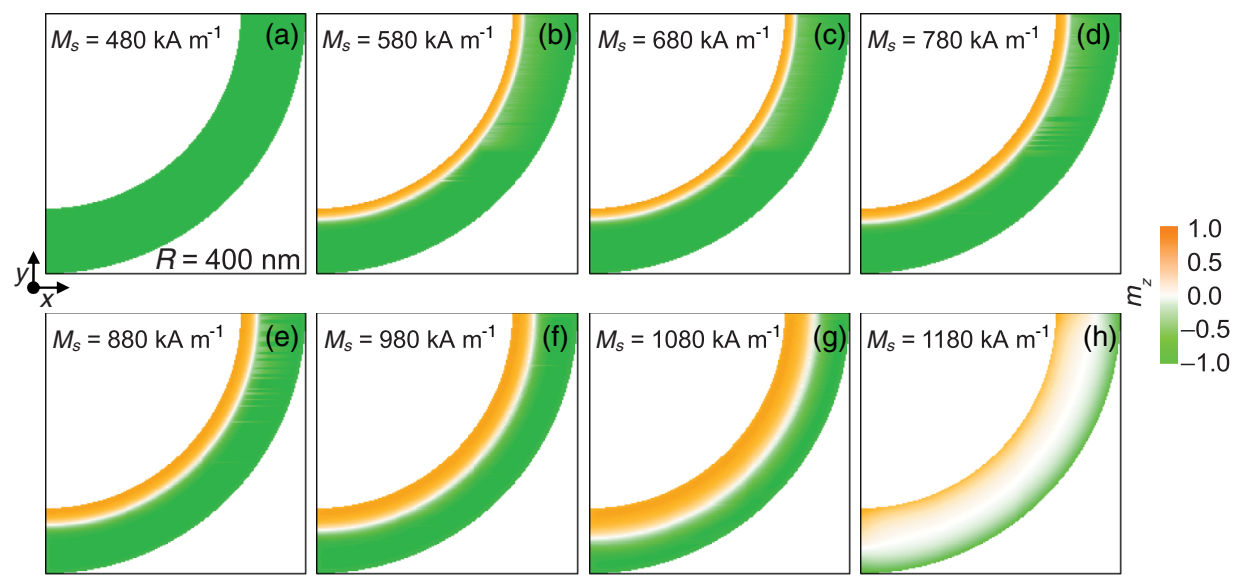

FIG. 15. Static domain patterns in a bent wire (1/4 arc) for various $M_{s}$ as indicated in (a)-(h). Other parameters are fixed at $A=15 \mathrm{pJm}^{-1}, K_{u}=$ $0.8 \mathrm{MJm}^{-3}$, and $D=1.0 \mathrm{~mJ} \mathrm{~m}^{-2}$. The width and outer radius of the wire are 100 and $400 \mathrm{~nm}$, respectively. 


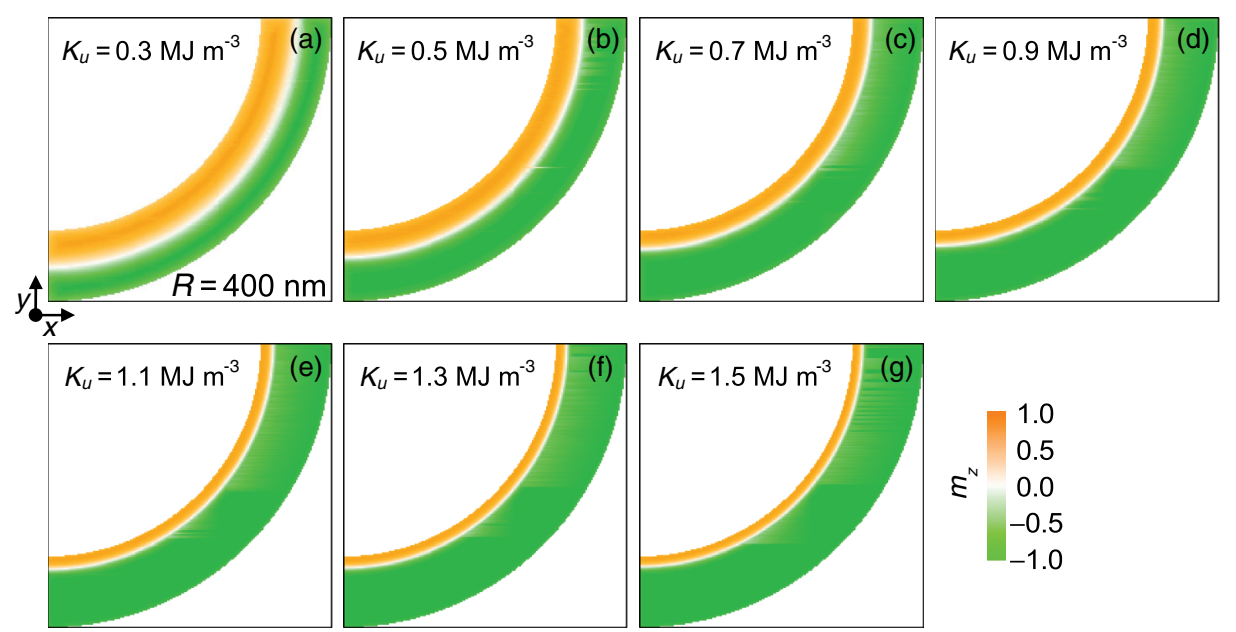

FIG. 16. Static domain patterns in a bent wire $(1 / 4 \mathrm{arc})$ for various $K_{u}$ as indicated in (a)-(g). Other parameters are fixed at $A=15 \mathrm{pJm}^{-1}$, $M_{s}=580 \mathrm{kAm}^{-1}$, and $D=2.0 \mathrm{mJm}^{-2}$. The width and outer radius of the wire are 100 and $400 \mathrm{~nm}$, respectively.
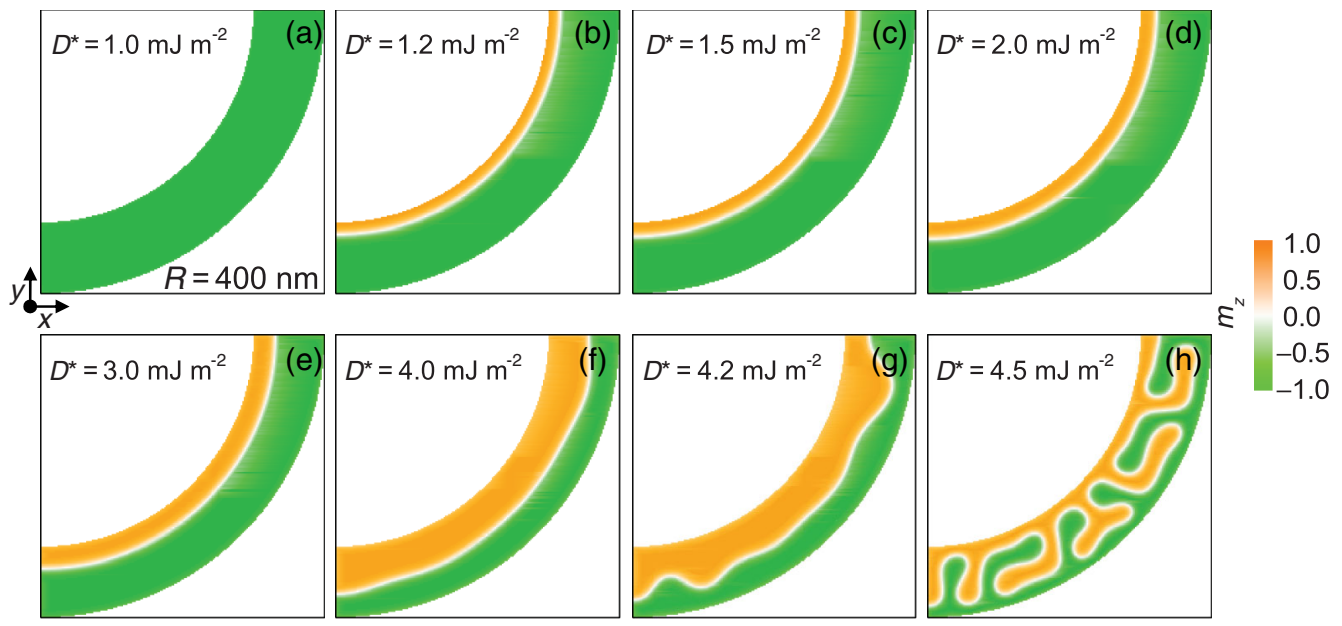

FIG. 17. Static domain patterns in a bent wire (1/4 arc) for various $D$ as indicated in (a)-(h). Other parameters are fixed at $A=$ $15 \mathrm{pJm}^{-1}, M_{s}=580 \mathrm{kAm}^{-1}$, and $K_{u}=0.8 \mathrm{MJm}^{-3}$. The width and outer radius of the wire are 100 and $400 \mathrm{~nm}$,
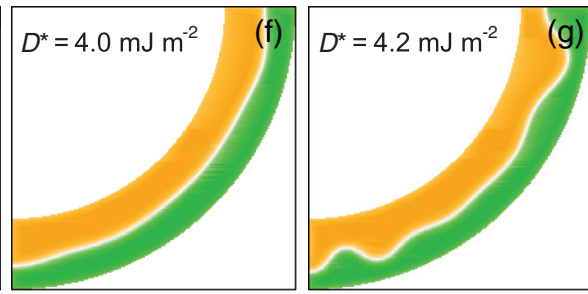
respectively.
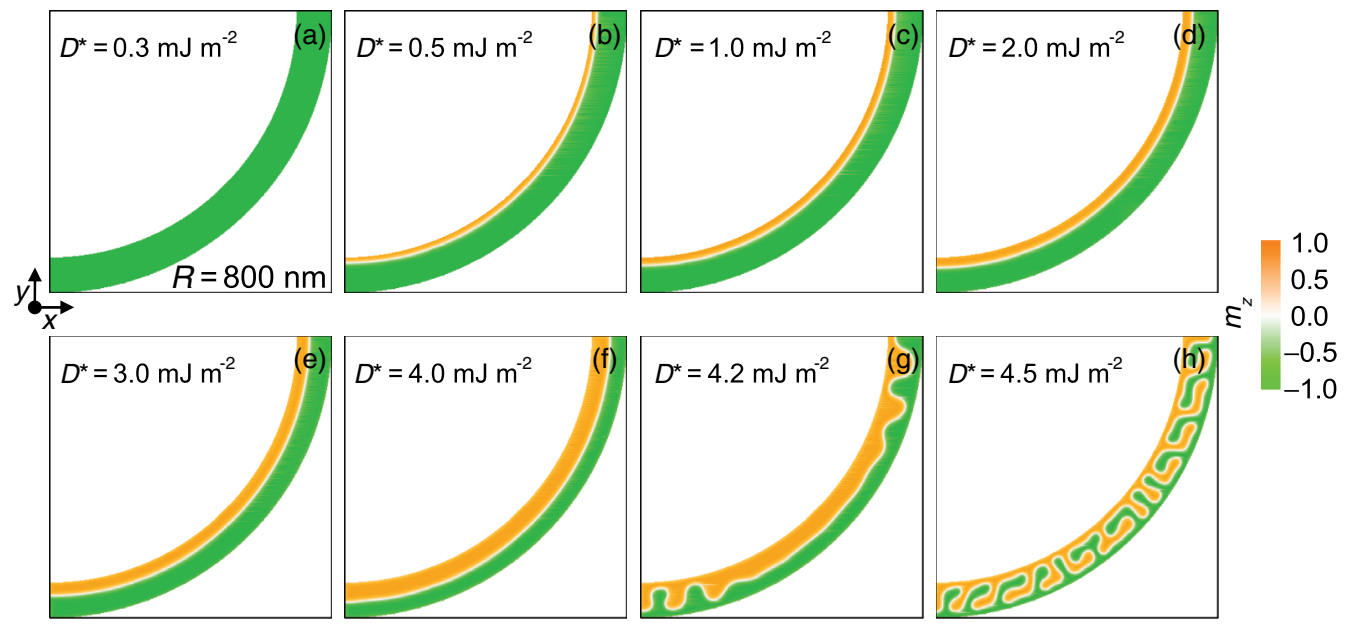

FIG. 18 Static domain patterns in a bent wire (1/4 arc) with an increased outer radius of $800 \mathrm{~nm}$ for various $D$ as indicated in (a)-(h). Other parameters are fixed at $A=15 \mathrm{pJm}^{-1}$, $M_{s}=580 \mathrm{kAm}^{-1}$, and $K_{u}=$ $0.8 \mathrm{MJm}^{-3}$. The width of the wire is $100 \mathrm{~nm}$.

lower threshold value $\left(D_{l}{ }^{*}\right)$ of $D$ required to stabilize a SDW decreases as the radius increases (compare Figs. 17-19), and for the 1200-nm arc, $D_{l}^{*}$ is close to zero (approximately $0.08 \mathrm{~mJ} \mathrm{~m}^{-2}$; see Fig. 19). This tendency is consistent with that for straight wires $[23,24]$, which are equivalent to arcs with an infinite radius.
Surprisingly, the upper threshold values $\left(D_{u}{ }^{*}\right)$ are almost independent of the arc radii (compare Figs. 17-19).

Detailed knowledge of SDW stability as a function of $K_{u}$ and $D$ is useful for device designs, considering that $K_{u}$ and $D$ are highly sensitive to the interface and layer thickness $[38,51,52]$. We, thus, derive the phase diagram 


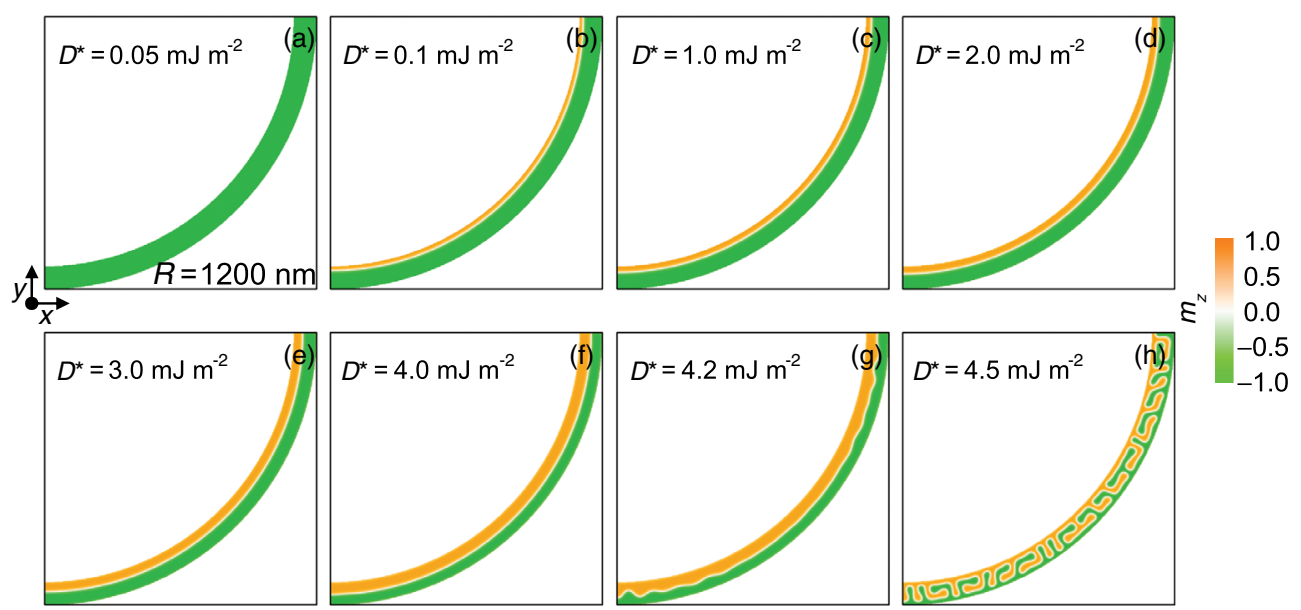

FIG. 19. Static domain patterns in a bent wire $(1 / 4$ arc) with an increased outer radius of $1200 \mathrm{~nm}$ for various $D$ as indicated in (a)-(h). Other parameters are fixed at $A=$ $15 \mathrm{pJ} \mathrm{m}^{-1}, M_{s}=580 \mathrm{kA} \mathrm{m}^{-1}$, and $K_{u}=0.8 \mathrm{MJ} \mathrm{m}^{-3}$. The width of the wire is $100 \mathrm{~nm}$.
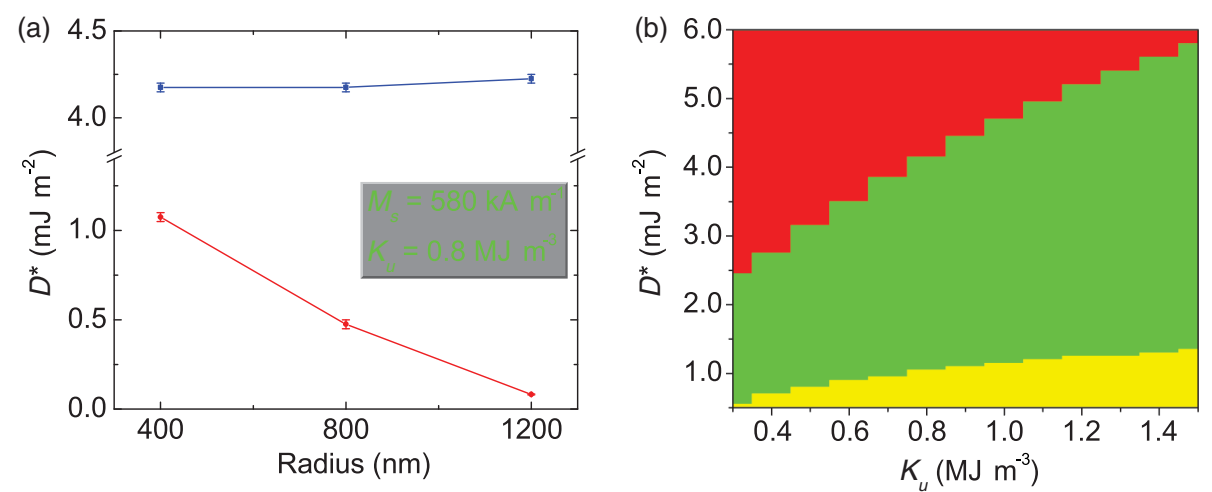

FIG. 20. (a) Critical IDMI strengths $\left(D^{*}\right)$ versus the outer radius of a bent wire $(1 / 4 \operatorname{arc}) . w=100 \mathrm{~nm}, A=15 \mathrm{pJ} \mathrm{m}^{-1}$, $M_{s}=580 \mathrm{kA} \mathrm{m}^{-1}$, and $K_{u}=0.8 \mathrm{MJ} \mathrm{m}^{-3}$. Square and circular dots denote the upper $\left(D_{u}{ }^{*}\right)$ and lower $\left(D_{l}{ }^{*}\right)$ critical values, respectively. Error bars are marked along with data points. Apparently, the smaller the bent wire is, the higher the $D_{l}{ }^{*}$. A SDW can be stabilized at static equilibrium when $D_{l}{ }^{*}<D<D_{u}{ }^{*}$. (b) Phase diagram for static magnetization states in a bent wire (1/4 arc) in the $K_{u}-D$ space. $R=400 \mathrm{~nm}, w=100 \mathrm{~nm}, A=15 \mathrm{pJ} \mathrm{m}^{-1}$, and $M_{s}=580 \mathrm{kA} \mathrm{m}^{-1}$. The bottom, middle, and top regions correspond to single-domain, SDW, and multidomain configurations, respectively, and the characteristic domain patterns are those in Figs. 17(a), 17(d), and 17(h).

for static spin configurations in the 400-nm arc in $K_{u}-D$ space, as shown in Fig. 20(b). Based on this plot, it is clear that the larger $K_{u}$, the wider the $D$ window in which a SDW can be stabilized. For realistic values of $D$, such as $D=1.0 \mathrm{~mJ} \mathrm{~m}^{-2}, K_{u}$ must be lower than a critical value to give a static SDW. For medium $D$ values, like $D=2.0 \mathrm{~mJ} \mathrm{~m}^{-2}$, SDWs are stable throughout the entire range of $K_{u}$, and for larger $D$ values, such as $D=4.0 \mathrm{~mJ} \mathrm{~m}^{-2}, K_{u}$ must be large enough to maintain a SDW. These points can act as a guide to choosing appropriate materials for device applications. Table I summarizes the static spin configurations for several special combinations of $K_{u}$ and $D$, with $M_{s}$ varying from 80 to $1580 \mathrm{kA} \mathrm{m}^{-1}$. These results reveal that a SDW can

TABLE I. Static magnetization states in a bent wire $(1 / 4 \mathrm{arc})$ as a function of $M_{s}, K_{u}$, and $D$. The units of $M_{s}, K_{u}$, and $D$ are kA m ${ }^{-1}$, $\mathrm{MJ} \mathrm{m}^{-3}$, and $\mathrm{mJ} \mathrm{m}^{-2}$, respectively. The numbers 0,1 , and 2 represent the single-domain, SDW, and multidomain states, respectively. $A=15 \mathrm{pJ} \mathrm{m}^{-1}, R=400 \mathrm{~nm}$, and $w=100 \mathrm{~nm}$.

\begin{tabular}{|c|c|c|c|c|c|c|c|c|c|c|c|c|c|c|c|c|}
\hline \multicolumn{17}{|c|}{$M_{s}$} \\
\hline$\left(K_{u}, D\right)$ & 80 & 180 & 280 & 380 & 480 & 580 & 680 & 780 & 880 & 980 & 1080 & 1180 & 1280 & 1380 & 1480 & 1580 \\
\hline$(0.8,1.0)$ & 0 & 0 & 0 & 0 & 0 & 1 & 1 & 1 & 1 & 1 & 1 & 2 & 2 & 2 & 2 & 2 \\
\hline$(0.8,3.5)$ & 1 & 1 & 1 & 1 & 1 & 1 & 1 & 1 & 2 & 2 & 2 & 2 & 2 & 2 & 2 & 2 \\
\hline$(1.4,1.0)$ & 0 & 0 & 0 & 0 & 0 & 0 & 0 & 0 & 1 & 1 & 1 & 1 & 1 & 1 & 1 & 2 \\
\hline$(1.4,3.5)$ & 1 & 1 & 1 & 1 & 1 & 1 & 1 & 1 & 1 & 1 & 1 & 1 & 1 & 2 & 2 & 2 \\
\hline
\end{tabular}


be stabilized in an arc (a bent wire) over a wide region of the parameter space, which is highly desirable for practical applications of SDW-based magnonic devices [65].

[1] V. V. Kruglyak, S. O. Demokritov, and D. Grundler, Magnonics, J. Phys. D 43, 264001 (2010).

[2] A. Chumak, V. Vasyuchka, A. Serga, and B. Hillebrands, Magnon spintronics, Nat. Phys. 11, 453 (2015).

[3] B. Lenk, H. Ulrichs, F. Garbs, and M. Munzenberg, The building blocks of magnonics, Phys. Rep. 507, 107 (2011).

[4] S. Bonetti and J. Åkerman, Magnonics: Nano-contact spintorque oscillators as magnonic building blocks, Top. Appl. Phys. 125, 177 (2013).

[5] M. M. Waldrop, The chips are down for Moore's law, Nature (London) 530, 144 (2016).

[6] InternationalTechnology Roadmap for Semiconductors, "Emerging Research Devices," 2009, http://www.itrs2.net/ itrs-reports.html.

[7] A. Khitun, M. Bao, and K. L. Wang, Magnonic logic circuits, J. Phys. D 43, 264005 (2010).

[8] A. Khitun, Magnonic holographic devices for special type data processing, J. Appl. Phys. 113, 164503 (2013).

[9] G. Csaba, A. Papp, and W. Porod, Spin-wave based realization of optical computing primitives, J. Appl. Phys. 115, 17C741 (2014).

[10] A. Kozhevnikov, F. Gertz, G. Dudko, Y. Filimonov, and A. Khitun, Pattern recognition with magnonic holographic memory device, Appl. Phys. Lett. 106, 142409 (2015).

[11] K. Vogt, F. Fradin, J. Pearson, T. Sebastian, S. Bader, B. Hillebrands, A. Hoffmann, and H. Schultheiss, Realization of a spin-wave multiplexer, Nat. Commun. 5, 3727 (2014).

[12] S. Urazhdin, V. E. Demidov, H. Ulrichs, T. Kendziorczyk, T. Kuhn, J. Leuthold, G. Wilde, and S. O. Demokritov, Nanomagnonic devices based on the spin-transfer torque, Nat. Nanotechnol. 9, 509 (2014).

[13] R. K. Dumas and J. Åkerman, Channeling spin waves, Nat. Nanotechnol. 9, 503 (2014).

[14] M. P. Kostylev, A. A. Serga, T. Schneider, B. Leven, and B. Hillebrands, Spin-wave logical gates, Appl. Phys. Lett. 87, 153501 (2005).

[15] V. E. Demidov, M. P. Kostylev, K. Rott, P. Krzysteczko, G. Reiss, and S. O. Demokritov, Excitation of microwaveguide modes by a stripe antenna, Appl. Phys. Lett. 95, 112509 (2009).

[16] M. Madami, S. Bonetti, S. Tacchi, G. Carlotti, G. Gubbiotti, G. Consolo, F. B. Mancoff, M. A. Yar, and Johan Åkerman, Direct observation of a propagating spin wave induced by spin transfer torque, Nat. Nanotechnol. 6, 635 (2011).

[17] M. Madami, E. Iacocca, S. R. Sani, G. Gubbiotti, S. Tacchi, R. K. Dumas, J. Åkerman, and G. Carlotti, Propagating spin waves excited by spin-transfer torque: A combined electrical and optical study, Phys. Rev. B 92, 024403 (2015).

[18] A. Houshang, E. Iacocca, P. Dürrenfeld, S. R. Sani, J. Åkerman, and R. K. Dumas, Spin-wave-beam driven synchronization of nanocontact spin-torque oscillators, Nat. Nanotechnol. 11, 280 (2016).
[19] J. Topp, J. Podbielski, D. Heitmann, and D. Grundler, Internal spin-wave confinement in magnetic nanowires due to zig-zag shaped magnetization, Phys. Rev. B 78, 024431 (2008).

[20] G. Duerr, K. Thurner, J. Topp, R. Huber, and D. Grundler, Enhanced Transmission through Squeezed Modes in a SelfCladding Magnonic Waveguide, Phys. Rev. Lett. 108, 227202 (2012).

[21] K. Vogt, H. Schultheiss, S. Jain, J. E. Pearson, A. Hoffmann, S. D. Bader, and B. Hillebrands, Spin waves turning a corner, Appl. Phys. Lett. 101, 042410 (2012).

[22] F. Garcia-Sanchez, P. Borys, R. Soucaille, J.-P. Adam, R. L. Stamps, and J.-V. Kim, Narrow Magnonic Waveguides Based on Domain Walls, Phys. Rev. Lett. 114, 247206 (2015).

[23] X. Xing and Y. Zhou, Fiber optics for spin waves, NPG Asia Mater. 8, e246 (2016).

[24] K. Wagner, A. Kákay, K. Schultheiss, A. Henschke, T. Sebastian, and H. Schultheiss, Magnetic domain walls as reconfigurable spin-wave nanochannels, Nat. Nanotechnol. 11, 432 (2016).

[25] D. Grundler, Spintronics: Nanomagnonics around the corner, Nat. Nanotechnol. 11, 407 (2016).

[26] A SDW is the boundary between two strip domains that have different magnetization orientations. From the structural viewpoint, a SDW differs from a conventional domain wall in the sense that it is elongated along the domain-wall surface. Usually, a conventional domain wall is orthogonal to the length of a magnetic wire and across the entire width of the wire. By contrast, a SDW is along the length of a magnetic wire and will not overspread the entire length of the wire, unless the injection process is finished. When a SDW is expanding, it includes a linear SDW ridge and a bent SDW head.

[27] See Supplemental Material at http://link.aps.org/ supplemental/10.1103/PhysRevApplied.7.054016 for the SDW-injection processes achieved in the Y-shaped nanostructure under various conditions.

[28] J. E. Hirsch, Spin Hall Effect, Phys. Rev. Lett. 83, 1834 (1999).

[29] P. P. J. Haazen, E. Murè, J. H. Franken, R. Lavrijsen, H. J. M. Swagten, and B. Koopmans, Domain wall depinning governed by the spin Hall effect, Nat. Mater. 12, 299 (2013).

[30] S. Emori, U. Bauer, S.-M. Ahn, E. Martinez, and G. S. D. Beach, Current-driven dynamics of chiral ferromagnetic domain walls, Nat. Mater. 12, 611 (2013).

[31] K.-S. Ryu, L. Thomas, S.-H. Yang, and S. Parkin, Chiral spin torque at magnetic domain walls, Nat. Nanotechnol. 8, 527 (2013).

[32] W. Jiang, P. Upadhyaya, W. Zhang, G. Yu, M. B. Jungfleisch, F. Y. Fradin, J. E. Pearson, Y. Tserkovnyak, K. L. Wang, O. Heinonen, S. G. E. te Velthuis, and A. Hoffmann, Blowing magnetic Skyrmion bubbles, Science 349, 283 (2015).

[33] S. Woo, K. Litzius, B. Krüger, M.-Y. Im, L. Caretta, K. Richter, M. Mann, A. Krone, R. M. Reeve, M. Weigand, P. Agrawal, I. Lemesh, M.-A. Mawass, P. Fischer, M. Kläui, and G. S. D. Beach, Observation of room-temperature magnetic Skyrmions and their current-driven dynamics in ultrathin metallic ferromagnets, Nat. Mater. 15, 501 (2016). 
[34] Experimentally, the oxide layer can be formed by oxidizing the HM2 material [32,35] or other metallic materials [36-38] deposited on a FM layer or by directly depositing a dielectric oxide [30] on a FM layer.

[35] G. Yu, P. Upadhyaya, Y. Fan, J. G. Alzate, W. Jiang, K. L. Wong, S. Takei, S. A. Bender, L.-T. Chang, Y. Jiang, M. Lang, J. Tang, Y. Wang, Y. Tserkovnyak, P. K. Amiri, and K. L. Wang, Switching of perpendicular magnetization by spin-orbit torques in the absence of external magnetic fields, Nat. Nanotechnol. 9, 548 (2014).

[36] K. Garello, I. M. Miron, C. O. Avci, F. Freimuth, Y. Mokrousov, S. Blugel, S. Auffret, O. Boulle, G. Gaudin, and P. Gambardella, Symmetry and magnitude of spin-orbit torques in ferromagnetic heterostructures, Nat. Nanotechnol. 8, 587 (2013).

[37] O. Boulle, J. Vogel, H. Yang, S. Pizzini, D. de Souza Chaves, A. Locatelli, T. O. Menteş, A. Sala, L. D. BudaPrejbeanu, O. Klein, M. Belmeguenai, Y. Roussigné, A. Stashkevich, S. M. Chérif, L. Aballe, M. Foerster, M. Chshiev, S. Auffret, I. M. Miron, and G. Gaudin, Roomtemperature chiral magnetic Skyrmions in ultrathin magnetic nanostructures, Nat. Nanotechnol. 11, 449 (2016).

[38] J. Cho, N.-H. Kim, S. Lee, J.-S. Kim, R. Lavrijsen, A. Solignac, Y. Yin, D.-S. Han, N. J. J. van Hoof, H. J. M. Swagten, B. Koopmans, and C.-Y. You, Thickness dependence of the interfacial Dzyaloshinskii-Moriya interaction in inversion symmetry broken systems, Nat. Commun. 6, 7635 (2015).

[39] A. Fert, Magnetic and transport properties of metallic multilayers, Mater. Sci. Forum 59-60, 439 (1990).

[40] M. I. Dyakonov and V. I. Perel, Current-induced spin orientation of electrons in semiconductors, Phys. Lett. 35A, 459 (1971).

[41] K. Ando, S. Takahashi, K. Harii, K. Sasage, J. Ieda, S. Maekawa, and E. Saitoh, Electric Manipulation of Spin Relaxation Using the Spin Hall Effect, Phys. Rev. Lett. 101, 036601 (2008).

[42] S. Zhang and Z. Li, Roles of Nonequilibrium Conduction Electrons on the Magnetization Dynamics of Ferromagnets, Phys. Rev. Lett. 93, 127204 (2004).

[43] A. Thiaville, Y. Nakatani, J. Miltat, and Y. Suzuki, Micromagnetic understanding of current-driven domain wall motion in patterned nanowires, Europhys. Lett. 69, 990 (2005).

[44] Actually, inserting the insulating layer is not essential because the SDW injection is not significantly influenced by the Zhang-Li torques accompanying the SHT; see Appendix A.

[45] A. Brataas, A. D. Kent, and H. Ohno, Current-induced torques in magnetic materials, Nat. Mater. 11, 372 (2012).

[46] A. V. Khvalkovskiy, V. Cros, D. Apalkov, V. Nikitin, M. Krounbi, K. A. Zvezdin, A. Anane, J. Grollier, and A. Fert, Matching domain-wall configuration and spin-orbit torques for efficient domain-wall motion, Phys. Rev. B 87, 020402 (2013).

[47] A. Manchon and S. Zhang, Theory of nonequilibrium intrinsic spin torque in a single nanomagnet, Phys. Rev. B 78, 212405 (2008).

[48] J. C. Slonczewski, Current-driven excitation of magnetic multilayers, J. Magn. Magn. Mater. 159, L1 (1996).
[49] S. Zhang, P. M. Levy, and A. Fert, Mechanisms of SpinPolarized Current-Driven Magnetization Switching, Phys. Rev. Lett. 88, 236601 (2002).

[50] M. R. Scheinfein, LLG Micromagnetics Simulator (release LLG v4_x64), http://llgmicro.home.mindspring.com/.

[51] C. Moreau-Luchaire, C. Moutafis, N. Reyren, J. Sampaio, C. A. F. Vaz, N. Van Horne, K. Bouzehouane, K. Garcia, C. Deranlot, P. Warnicke, P. Wohlhüter, J.-M. George, M. Weigand, J. Raabe, V. Cros, and A. Fert, Additive interfacial chiral interaction in multilayers for stabilization of small individual Skyrmions at room temperature, Nat. Nanotechnol. 11, 444 (2016).

[52] G. Chen, T. Ma, A. T. N'Diaye, H. Kwon, C. Won, Y. Wu, and A.K. Schmid, Tailoring the chirality of magnetic domain walls by interface engineering, Nat. Commun. 4, 2671 (2013).

[53] J. Sampaio, V. Cros, S. Rohart, A. Thiaville, and A. Fert, Nucleation, stability and current-induced motion of isolated magnetic Skyrmions in nanostructures, Nat. Nanotechnol. 8, 839 (2013).

[54] W. Jiang, X. Zhang, G. Yu, W. Zhang, M. B. Jungfleisch, J. E. Pearson, O. Heinonen, K. L. Wang, Y. Zhou, A. Hoffmann, and S. G. E. te Velthuis, Direct observation of the Skyrmion Hall effect, Nat. Phys. 13, 162 (2016).

[55] L. Liu, C.-F. Pai, Y. Li, H. W. Tseng, D. C. Ralph, and R. A. Buhrman, Spin-torque switching with the giant spin Hall effect of tantalum, Science 336, 555 (2012).

[56] O. Krupin, G. Bihlmayer, K. Starke, S. Gorovikov, J. E. Prieto, K. Döbrich, S. Blügel, and G. Kaindl, Rashba effect at magnetic metal surfaces, Phys. Rev. B 71, 201403(R) (2005).

[57] H. Cercellier, C. Didiot, Y. Fagot-Revurat, B. Kierren, L. Moreau, D. Malterre, and F. Reinert, Interplay between structural, chemical, and spectroscopic properties of $\mathrm{Ag} / \mathrm{Au}(111)$ epitaxial ultrathin films: A way to tune the Rashba coupling, Phys. Rev. B 73, 195413 (2006).

[58] J. Iwasaki, M. Mochizuki, and N. Nagaosa, Current-induced Skyrmion dynamics in constricted geometries, Nat. Nanotechnol. 8, 742 (2013).

[59] Y. Zhou and M. Ezawa, A reversible conversion between a Skyrmion and a domain-wall pair in a junction geometry, Nat. Commun. 5, 4652 (2014).

[60] H. Y. Yuan and X. R. Wang, Boosting domain wall propagation by notches, Phys. Rev. B 92, 054419 (2015).

[61] E. Iacocca, R. K. Dumas, L. Bookman, M. Mohseni, S. Chung, M. A. Hoefer, and J. Åkerman, Confined Dissipative Droplet Solitons in Spin-Valve Nanowires with Perpendicular Magnetic Anisotropy, Phys. Rev. Lett. 112, 047201 (2014).

[62] X. Xing, P. W. T. Pong, and Y. Zhou, Current-controlled unidirectional Edge-Meron motion, J. Appl. Phys. 120, 203903 (2016).

[63] S.-Z. Lin, Edge instability in a chiral stripe domain under an electric current and Skyrmion generation, Phys. Rev. B 94, 020402(R) (2016).

[64] This confining force originates from the variation in the total potential energy of the magnetic system with respect to the SDW expansion. There is no such force after the SDW head reaches the other end or before the SDW head is nucleated. 
[65] J. Lan, W. Yu, R. Wu, and J. Xiao, Spin-Wave Diode, Phys. Rev. X 5, 041049 (2015).

[66] X. Xing, S. Li, X. Huang, and Z. Wang, Engineering spinwave channels in submicrometer magnonic waveguides, AIP Adv. 3, 032144 (2013).

[67] X. Xing, Y. Yu, S. Li, and X. Huang, How do spin waves pass through a bend?, Sci. Rep. 3, 2958 (2013).

[68] X. Xing, W. Yin, and Z. Wang, Excitation of antisymmetric modes and modulated propagation of spin waves in bent magnonic waveguides, J. Phys. D 48, 215004 (2015).

[69] K. Y. Guslienko, S. O. Demokritov, B. Hillebrands, and A. N. Slavin, Effective dipolar boundary conditions for dynamic magnetization in thin magnetic stripes, Phys. Rev. B 66, 132402 (2002).

[70] P. Clausen, K. Vogt, H. Schultheiss, S. Schäfer, B. Obry, G. Wolf, P. Pirro, B. Leven, and B. Hillebrands, Mode conversion by symmetry breaking of propagating spin waves, Appl. Phys. Lett. 99, 162505 (2011).

[71] V. E. Demidov, S. O. Demokritov, K. Rott, P. Krzysteczko, and G. Reiss, Mode interference and periodic self-focusing of spin waves in permalloy microstripes, Phys. Rev. B 77, 064406 (2008).

[72] M. P. Kostylev, G. Gubbiotti, J.-G. Hu, G. Carlotti, T. Ono, and R. L. Stamps, Dipole-exchange propagating spin-wave modes in metallic ferromagnetic stripes, Phys. Rev. B 76, 054422 (2007).

[73] F. Garcia-Sanchez, P. Borys, A. Vansteenkiste, J. V. Kim, and R. L. Stamps, Nonreciprocal spin-wave channeling along textures driven by the Dzyaloshinskii-Moriya interaction, Phys. Rev. B 89, 224408 (2014).

[74] V. E. Demidov, S. O. Demokritov, K. Rott, P. Krzysteczko, and G. Reiss, Nano-optics with spin waves at microwave frequencies, Appl. Phys. Lett. 92, 232503 (2008).

[75] I. M. Miron, G. Gaudin, S. Auffret, B. Rodmacq, A. Schuhl, S. Pizzini, J. Vogel, and P. Gambardella, Current-driven spin torque induced by the Rashba effect in a ferromagnetic metal layer, Nat. Mater. 9, 230 (2010).

[76] M. Dvornik, Y. Au, and V. V. Kruglyak, Magnonics: Micromagnetic simulations in magnonics, Top. Appl. Phys. 125, 101 (2013).

[77] M. Heide, G. Bihlmayer, and S. Blügel, DzyaloshinskiiMoriya interaction accounting for the orientation of magnetic domains in ultrathin films: Fe/W(110), Phys. Rev. B 78, 140403 (2008).

[78] M. A. W. Schoen, D. Thonig, M. L. Schneider, T. J. Silva, H. T. Nembach, O. Eriksson, O. Karis, and J. M. Shaw, Ultra-low magnetic damping of a metallic ferromagnet, Nat. Phys. 12, 839 (2016).

[79] O. d'Allivy Kelly, A. Anane, R. Bernard, J. B. Youssef, C. Hahn, A. H. Molpeceres, C. Carrétéro, E. Jacquet, C. Deranlot, P. Bortolotti, R. Lebourgeois, J.-C. Mage, G. de Loubens, O. Klein, V. Cros, and A. Fert, Inverse spin Hall effect in nanometer-thick yttrium iron garnet/Pt system, Appl. Phys. Lett. 103, 082408 (2013).

[80] M. C. Onbasli, A. Kehlberger, D. H. Kim, G. Jakob, M. Kläui, A. V. Chumak, B. Hillebrands, and C. A. Ross, Pulsed laser deposition of epitaxial yttrium iron garnet films with low Gilbert damping and bulk-like magnetization, APL Mater. 2, 106102 (2014).

[81] In addition to 0.02 , we also test other damping values, 0.01 , 0.005 , and 0.001 , for which the injection processes do not distinguish themselves from each other.

[82] A. A. Serga, A. V. Chumak, and B. Hillebrands, YIG magnonics, J. Phys. D 43, 264002 (2010).

[83] H. Ulrichs, V. E. Demidov, S. O. Demokritov, W. L. Lim, J. Melander, N. Ebrahim-Zadeh, and S. Urazhdin, Optimization of Pt-based spin-Hall-effect spintronic devices, Appl. Phys. Lett. 102, 132402 (2013).

[84] A. A. Thiele, Steady-State Motion of Magnetic Domains, Phys. Rev. Lett. 30, 230 (1973). 